\title{
Entry of Not-for-Profit Food Cooperatives and Its Implications on For-Profit Retailers
}

\author{
C. Gizem Korpeoglu \\ Department of Industrial Engineering and Innovation Sciences, Eindhoven University of Technology, Eindhoven, Netherlands, \\ 5600 MB, c.g.korpeoglu@tue.nl \\ Ersin Körpeoğlu \\ School of Management, University College London, London, UK, E14 5AA, e.korpeoglu@ucl.ac.uk \\ Christopher S. Tang \\ UCLA Anderson School of Management, University of California, Los Angeles, Los Angeles, California 90095, \\ chris.tang@anderson.ucla.edu \\ Jiayi Joey $\mathrm{Yu}^{*}$ \\ School of Management, Fudan University, Shanghai 200433, China, yujiayi@fudan.edu.cn
}

\begin{abstract}
Propelled by the recent social activism that calls for not-for-profit food cooperatives (coops) to address the food desert and job desert issues, more food coops are establishing their presence in less-populated areas or poor communities. This trend has motivated us to examine the entry conditions for food coops with the following two related social missions: (A) maximize reserve to support the local community needs; and (B) maximize sales to support the local economy. We present a game-theoretic model to analyze the competition between an entrant not-for-profit coop and an incumbent for-profit retailer in a market comprising heterogeneous consumers with different annual consumption rates and social benefit levels. We examine the coop's pricing strategy and entry conditions, the impact of the coop's entry on the retailer's profit, and the conditions under which the retailer should deter the coop's entry. Our analytical results indicate that a coop can afford to enter the market only when its fixed annual operating cost is below a certain threshold. Upon entry, it is optimal for the coop to set a membership fee and a member-only discount to attract at least those consumers with high consumption rate. We show that the coop's entry is detrimental for the retailer. However, interestingly, even if the retailer can profitably deter the coop's entry, it is actually optimal for the retailer to tolerate it when the coop's annual fixed operating cost is below a threshold, where this threshold is lower for a coop with mission (B) than mission (A).
\end{abstract}

Key words: Food Deserts, Food Cooperatives, Not-for-Profit Operations, Social Conscience, Social Mission.

\section{Introduction}

The COVID-19 pandemic has exacerbated an already severe food-access issue in the United States. Even before the pandemic, 23.5 million people in the US lived in "food deserts" with no or limited access to healthy and affordable food, and nearly half of them were also low-income people. Although some initiatives such as the 2010 National Healthy Food Financing Initiative offered tax

* Corresponding Author. 
incentives for for-profit retailers to provide more accessible and affordable food, these initiatives did not seem to work as they caused economic disruptions in poor neighborhoods (Karpyn et al. 2019). These factors along with the chronic racial/social injustice in the US have stimulated the entry of not-for-profit food coops, facilitating access to affordable and high-quality food while supporting neighborhood well-being (e.g., Nembhard 2014b, Halliday and Foster 2020). Yet, this social movement of food coops may trigger existing for-profit retailers to deter their entry or compete more fiercely (Picchi 2015). Thus, in this paper, we analyze the entry conditions and pricing decisions of not-for-profit food coops and investigate when coops can co-exist with for-profit retailers.

We primarily focus on food coops that support access to healthy food in under-served areas through community building such as economic development and job creation for the local community (Halliday and Foster 2020). Specifically, we analyze member-owned food coops that operate according to 7 Rochdale Principles, ${ }^{1}$ provide access to affordable healthy food, support local producers, and revitalize the local economy (Knupfer 2013). Although some active food coops such as International Cooperative Alliance date back to the late nineteenth century, the entry of food coops tend to rise during a recession caused by a war or pandemic (Nargi 2020). As the world is facing an economic recession caused by the COVID-19 pandemic, the entry of food coops becomes a prominent topic of interest especially in the United States.

Recent establishment of food coops has been motivated by three factors: access to affordable and healthy food, local job creation, and the fight against racial injustices. First, food cooperatives are being established to provide the poor with access to affordable and healthy food in the United States (Wilbourn 2015). For example, Karpyn et al. (2019) reports that residents of low-income areas in New Jersey travel twice as far to access to food-retail locations as residents of middleincome areas. During the pandemic, reduced operating hours of grocers and reduced service of public transportation have made it even more challenging for the poor or the elderly to travel long distance to access fresh food. ${ }^{2}$ Without easy access to affordable fresh food, hunger and obesity ensue. Peters (2018) argues that food coops can improve access to fresh food, and Brinkley (2019) provide evidence that food coops are more effective solving food access problem than for-profit retailers (such as Walmart).

Second, new food cooperatives are established to support job creation in poor neighborhoods because most food deserts are also "job deserts" in the United States (Karpyn et al. 2019). For

${ }^{1}$ According to the International Cooperative Alliance, an NGO founded in 1895 with 1.2 billion cooperative members, these 7 Rochdale Principles are voluntary and open membership, democratic member control, member economic participation, autonomy and independence, education, training, and information, cooperation among cooperatives, and concern for community.

${ }^{2}$ Online grocery shopping is not available in many areas, but even when it is available, it is not equally accessible. In addition to unequal internet access, some deliveries have minimum purchase requirements or shipping fees, which can be a barrier for low-income customers. 
example, Berg (2011) reports that, in 2007, South Bronx in New York was a food desert with $13.9 \%$ unemployment and $35 \%$ able-bodied adults remained outside the workforce. To address these issues, more food coops with different social missions open in food desserts and adopt local hiring practices, pay higher wages, and help counteract inequities in the food system (Brinkley 2019).

Third, a growing number of food cooperatives are established to support the fight against racial injustices. Controlling for the poverty rate, Brooks (2014) shows that food deserts are more prevalent in black and hispanic neighborhoods than their white counterparts. For example, PCC Community Markets opened its Central District of Seattle store in 2020 to serve the black community by training its staff on implicit racial bias, recruiting members from the black community, and developing a career mapping program to ensure equitable advancement opportunity for the minority group (Seattle 2020). Besides Seattle, Philadelphia has witnessed the opening of new food coops in black neighborhoods recently (Brzyski 2019). ${ }^{3}$

The above three factors propel the entry of food coops and motivate our study. It is important to note that the entry of a food coop is different from the widely-studied entry of for-profit retailers primarily because food coops differ from for-profit retailers in three fundamental aspects:

1. Not-for-Profit. Cooperatives are usually not-for-profit organizations, and they are owned and operated by their members (not shareholders) to achieve certain economic and social missions. ${ }^{4}$

2. Social Missions. By reviewing the mission statement of over 50 food coops, we have identified two social missions commonly supported by food coops:

(A) Maximize reserve to support local community needs. Some food coops such as PCC Community Markets in Seattle and Co-operative Group in the United Kingdom use their reserve (created out of their profits) to support the local community activities such as discounted cooking classes. For example, Gulker (2018) reports that a food coop called Friends of Bob located in Lafayette, Indiana, sponsors and works with local venues to organize events such as musical concerts.

\footnotetext{
${ }^{3}$ Recognizing that food coops are primarily white-led, Adams (2020) calls for black-led food coops in food deserts to revitalize black neighborhoods. Opening in 2009, the black-led Mandela Foods Coop in West Oakland has provided fresh food and economic opportunities in West Oakland, and it is planning to open its second coop store in East Oakland in 2020. Roberts (2019) reports that the black-led coop movement is generating momentum. Some notable examples include (a) the 2020 opening of an African American-led Detroit People's Food Coop in North End, serving predominately African American in the low- and moderate-income community; and (b) the 2020 opening of Central Brooklyn Food Coop in the majority-black Bedford Stuyvesant and Crown Heights neighborhoods, offering affordable fresh food to customers and supporting black producers and distributors (Elliott 2020).

${ }^{4}$ There are a few cooperatives that operate as limited cooperative association (LCA) in the US around issues of investor control and tax implications (Deller et al. 2009). A not-for-profit cooperative corporation is different from a tax-exempt nonprofit corporation as follows: (1) A tax-exempt nonprofit organization cannot distribute profits to members, while a cooperative corporation can distribute its profits based on members' participation in the cooperative through patronage rebates (e.g., a food cooperative Fair Price of Singapore offers patronage rebates annually based on their purchases throughout the year). (2) A cooperative corporation generates most or all of its revenue through the sales of goods and services; however, a tax-exempt nonprofit organization can receive tax-deductible donations from community members and foundations, and is limited in the amount of business activity it conducts unrelated to its charitable purpose.
} 
(B) Maximize sales to support the local economy. Some food coops such as Electric City Food Cooperative in Schenectady, NY are committed to grow their sales for creating more jobs for the local community (Nembhard 2014a). Deller et al. (2009) reports that 15,000 jobs are supported by 290 food coops in the US. Nelson (2020) reports that most food coops are committed to sourcing from local producers to support the local economy. Specifically, they source from an average of 157 local farmers and producers, and an average food coop purchases $20 \%$ of the products it sells from local sources (Catlette 2012). Despite higher volumes, large for-profit food stores source far fewer local products than food coops, specifically, averaging 65 local producers, and $6 \%$ of sales.

3. Membership Fees and Member Privileges. Unlike most for-profit retailers, food coops usually charge a lump-sum or an annual membership fee, and all members are invited to vote on major initiatives. Also, most food coops are open to non-members as well so that both members and non-members can shop at the food coop (e.g., Fair Price in Singapore). However, members usually get "member-only" price discounts or rebates on certain items. ${ }^{5}$

These three distinct aspects not only differentiate a food coop from a for-profit retailer, but also lead to new operational complexities. ${ }^{6}$ Furthermore, as more not-for-profit food coops with different social missions enter the market, it is of interest to examine the impact of their entry on for-profit retailers and whether retailers would try to deter such entry. These observations motivate us to examine the following research questions arising from the entry of a not-for-profit coop with a social mission (A) maximize reserve to support local community needs or (B) maximize sales to support the local economy.

1. What are the entry conditions of a not-for-profit coop with a social mission (A) or (B) in the presence of an existing for-profit retailer? Upon entering the market, how should the coop set its membership fee, retail price, and member-only price discount?

2. From the incumbent retailer's perspective, what is the impact of the entry of a coop with a social mission (A) or (B) on the retailer's sales quantity and profit?

3. Should the retailer set a low retail price proactively to deter the coop's entry? Will the retailer be better off by tolerating the entry of the coop?

\footnotetext{
5 There are some "exclusive" food coops who serve only members. For example, Macomb Coop located in Macomb, Illinois, has a population of 20,000 members. This coop has developed an e-commerce program so that farmers and producers list their supply available for the next week, and members (who pay $\$ 100$ per year in membership fees) can place orders online and pick up their produce (Kauffman 2017). Also, by charging an annual "warehouse club" membership fee, Costco offers discounts by registering as a for-profit "wholesale club."

${ }^{6}$ Even though a coop with social mission A has a similar objective to that of a profit-maximizing retailer, a coop with social mission A fundamentally differs from a profit-maximizing retailer for two reasons. First, the social causes that the coop supports resonates positively in socially-conscious consumers, and creates added value for coop shoppers. Second, the coop uses the membership mechanism and offers membership privileges unlike most for-profit retailers with a few exceptions such as Costco. To the best of our knowledge, the entry of for-profit retailers with membership system has not been studied in the literature either.
} 
As an initial attempt to examine the above questions, we present a parsimonious model to capture the essence of a not-for-profit food coop with a social mission (A) or (B). To this end, we analyze a two-stage Stackelberg competition between an incumbent for-profit retailer and an entrant coop in a market comprised of consumers that are heterogenous in terms of their annual consumption (high or low) and "social benefit" levels (which can take continuous values). The social benefit captures unique values a consumer receives from buying from the coop such as the convenience of the food coop's location in a food desert, a sense of satisfaction from supporting local businesses, or a warm glow from contributing to the social causes that the coop supports (cf. Andreoni 1990 and Harbaugh 1998). By analyzing the strategic interactions among utility-maximizing consumers, a profit-maximizing retailer, and a not-for-profit coop with social mission (A) or (B), we obtain the following results.

We first analyze the coop's entry conditions and its competition with the retailer. We find that a coop with mission (A) or (B) can only afford to enter the market when consumers' social benefit levels are sufficiently high. This is somewhat intuitive. Perhaps more interestingly, high socialbenefit levels can also help the retailer survive against a coop with social mission (A), but it weakens the retailer's survival against the coop with social mission (B). ${ }^{7}$ In the latter case, the retailer and the coop can co-exist only when the coop's cost of entry (i.e., its fixed annual operating cost) takes moderate values. We further show that, when the coop is able to enter the market, it is optimal for the coop (regardless of its social mission) to set its membership fee and member-only discount to induce at least the high-consumption consumers to become members.

We next examine the impact of a coop's entry on the retailer, and whether the retailer should deter or tolerate the coop's entry. We find that a coop's entry is always detrimental to the retailer's profit. This is somewhat intuitive. Yet, more interestingly, we find that, even when a retailer can profitably deter the entry of a coop, it is optimal for the retailer to "tolerate" the coop's entry when the coop's cost of entry is below a certain threshold. We further find that the entry of a coop with social mission (B) is more detrimental to the retailer's profit than the entry of a coop with social mission (A), so that the retailer is more eager to deter the entry of the coop with social mission (B). Hence, our results indicate that a for-profit retailer is more likely to co-exist with a coop with social mission (A).

${ }^{7}$ A coop may be able to obtain locally-sourced products at a lower unit cost (due to lower transportation and storage costs), but the unit cost for national brand products is likely to be higher than for-profit retailers (due to the coop's smaller scale). However, because coops do not focus on generating profits for themselves, it may be possible for coops to sell their products at a lower retail price to support their social missions (A) and (B) as stated above. Consistent with our findings, Jackson (2019) reports that food coop retail prices can be lower or higher than supermarkets. According to a price comparison of 700 items conducted by the Community Food Coop based in Bellingham, Washington in 2018, the coop's retail price is 3.5\% lower (on average) across 402 items that are in common with Whole Foods and $2.7 \%$ lower (on average) across 259 items that are in common with Haggen, but it is $2.6 \%$ higher (on average) across 307 items that are in common with Fred Meyer (Jackson 2019). 


\section{Literature Review}

Although 290 food coops operating in the US account for $\$ 2.1$ billion in sales revenue, the literature on coops is scant (Deller et al. 2009). Due to an emerging interest in studying socially responsible operations in recent years, OM scholars have explored various issues arising from agricultural cooperatives. An et al. (2015) examine when farmers benefit from agricultural marketing cooperatives that can enable coops to sell their products as a single brand, and from agricultural supply cooperatives that can enable coops to sell directly to large companies such as Walmart. He et al. (2018) analyze farmer cooperatives using Cournot competition framework under asymmetric market information. Shi et al. (2019) study the optimal selling policies of farmer cooperatives for storable agricultural products under price fluctuations. Mu et al. (2019) examine how to eliminate free-riding in dairy cooperatives. Qian and Olsen (2020) explore the coordination of operational and financial decisions of an agricultural marketing cooperative called Frontera in New Zealand, where farmer members' equity is required to be in proportion to their patronage (i.e., produce supplied). Motivated by a for-profit olive oil cooperative in Turkey, Ayvaz-Cavdaroğlu et al. (2020) examine a quality-based payment to its risk-averse farmer members in order to incentivize them to invest in quality improvements so that their products can demand a higher market price. While the recent literature focuses on agricultural cooperatives, we examine food coops with two social missions. Not only is the issue we examine (i.e., entry strategy) different, but we also consider the competition between a not-for-profit coop and a for-profit retailer.

Our paper is also related to the market-entry literature pioneered by Bain (1949), who establishes the notion of an incumbent's decision to lower its price below profit maximizing price to deter the entry of a for-profit competitor. This literature mainly focuses on how a for-profit firm can deter a for-profit competitor, and suggests deterrence tools such as pricing (Bain 1949), strategic commitment (Spence 1977, 1979), long-term contracts (Aghion and Bolton 1987), cost signalling (Srinivasan 1991), bundle pricing (Nalebuff 2004), or discount contracts (Ide et al. 2016). There are also some other papers that extended the market entry problem by considering factors such as market uncertainty (Baron 1973) or information asymmetry (Milgrom and Roberts 1982). Overall these papers focus on the deterrence tools or the market structure rather than focusing on the entrant characteristics. (We refer the reader to Hall (2008) for a review of the market-entry literature.) More recently, Gao et al. (2017) examine the entry of copycats, and show that the incumbent can prevent the copycat from entering by selling a higher-quality product.

Our work contributes to the market entry literature on several fronts. First, unlike the literature that models the entry of for-profit retailers, we examine the entry of a not-for-profit coop with a social mission (A) or (B) into a market that is comprised of consumers with different social benefit levels. This social responsibility component adds a new dimension beyond the typical price 
competition. Furthermore, consistent with the practice of food cooperatives, our model equips the entrant with tools such as membership fees and discounts that are not covered in the market entry literature. All these factors allow our model to yield a richer analysis of market entry where the question of whether to deter entry or not is non-trivial. ${ }^{8}$ Finally, as another novel aspect, our results shed some light on how different social missions can affect the coop's membership fee, price, and member-only discount decisions and the retailer's deterrence strategy against the coop's entry.

\section{Model Preliminaries}

We consider a two-period Stackelberg competition in a local market between an incumbent (forprofit) retailer and an entrant (not-for-profit) food coop. The sequence of events, as depicted in Figure 1, is as follows. At the beginning of period 1, the retailer determines its retail price $r$ given a unit cost of $w_{r} \cdot{ }^{9}$ Upon observing the retailer's price $r$ and taking its own fixed annual operating cost $k$ (that includes rent and other fixed utility costs) into consideration, the coop decides whether to enter the local market at the beginning of period $2 .{ }^{10}$ If the coop enters, it makes three socialmission-specific decisions: the annual membership fee $F$, the retail price $p$, and the member-only discount $\delta$ (so that each member effectively pays $(p-\delta)$ per unit).

Upon observing the decisions of the retailer and the coop, each consumer shops at the store that minimizes her annual cost.

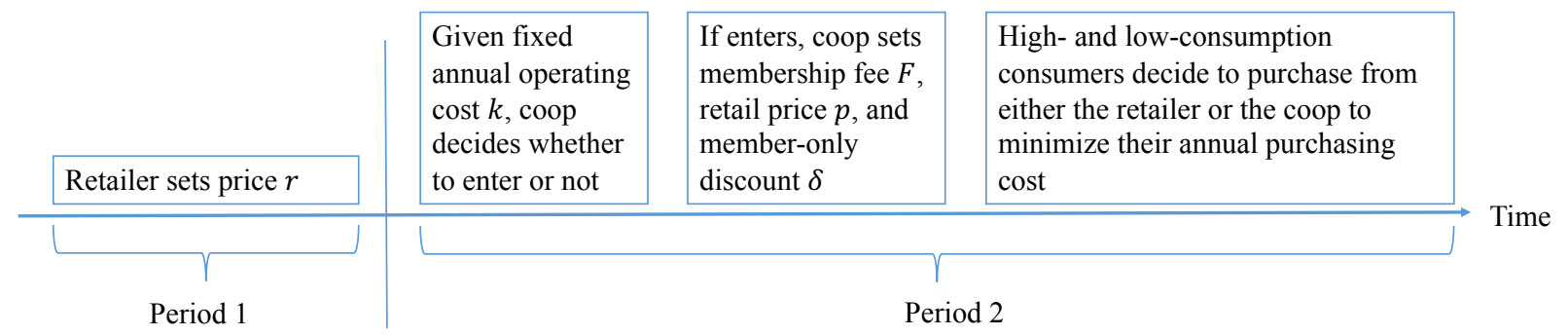

Figure 1 Sequence of events.

In our model, there is a continuum of consumers (with measure normalized to 1) in the local market with two different annual consumption rates. (That is akin to a two-point distribution.) A high-consumption type consumer consumes $h$ units per year, while a low-consumption type

${ }^{8}$ Although some papers in the supply chain competition literature analyze market entry (e.g., Corbett and Karmarkar 2001, Korpeoglu et al. 2020), these studies focus on the entry and competition of for-profit firms.

${ }^{9}$ As standard in a Stackelberg competition, we incorporate the notion of "irreversibility" of the retailer's retail price $r$. Spence (1977) articulates that irreversibility is a way for a firm to commit itself in advance to issue a credible threat to potential entry. For this reason and for tractability, we assume that the retailer does not change its retail price after the coop's entry. This assumption is commonly used in the market-entry literature (e.g., Gao et al. 2017) and is consistent with the notion of price stickiness (e.g., Chen et al. 2017).

${ }^{10}$ As we consider the coop as a new entrant, it seems reasonable to assume that the coop has a larger fixed annual operating cost than an established retailer. As we are more interested in the coop's entry conditions and the retailer's deterrence strategy, we normalize the pre-existing retailer's fixed annual operating cost to zero. 
consumer consumes $l$ units per year, where $h>l$. The proportions of the high- and low-consumption types are $\alpha$ and $1-\alpha$, respectively. ${ }^{11}$

When shopping at the retailer, each high-consumption consumer incurs an annual cost of $r h$ and each low-consumption consumer incurs an annual cost of $r l$. However, the annual cost for shopping at the coop is more involved. First, regardless of the consumption type, we assume that each consumer is endowed with a certain "social benefit" $S$, where $S$ captures unique values a consumer receives from shopping at the coop that include: the convenience of the food coop's location in a food desert, a sense of satisfaction from supporting local businesses, or a warm glow from contributing to the social causes that the coop supports (see §1). By using a modeling framework similar to the one presented in McCardle et al. (2009), we model social benefit $S$ as implicit "cost savings" when a consumer supports the coop through patronage. Second, to capture the heterogeneity across consumers, we assume that social benefit $S$ follows a uniform distribution such that $S \sim U[0, v]$, where we refer to the parameter $v$ as the "social-benefit level." By taking the implicit cost savings derived from the social benefit $S$ along with the coop's decisions (i.e., a fixed annual membership fee $F$, retail price $p$, and member-only discount $\delta$ ), the effective annual cost for a consumer (with high- or low-consumption, and with or without membership) are as summarized in Table 1.

Table 1 Effective annual cost of different types of consumers for shopping at the coop.

\begin{tabular}{c|c|c}
\hline & with membership & without membership \\
\hline high-consumption type & $F+(p-\delta) h-S$ & $p h-S$ \\
low-consumption type & $F+(p-\delta) l-S$ & $p l-S$ \\
\hline
\end{tabular}

\subsection{Sales and Profits under Different Coop Pricing Strategies}

By comparing the effective annual cost for shopping at the coop with and without membership as shown in Table 1, we can derive three coop-pricing strategies, each of which is associated with the annual membership fee $F$ and the member-only discount $\delta$ that is intended to induce certain types of consumers (with consumption $h$ or $l$ ) to become members if they choose to shop at the coop.

Strategy 1. Set $F \leq \delta l$ to induce both high- and low-consumption consumers to become members; Strategy 2. Set $\delta l \leq F \leq \delta h$ to induce only high-consumption consumers (but not low-consumption consumers) to become members; and

Strategy 3. Set $F \geq \delta h$ so that no consumers will become members.

We next determine the sales quantities and profits for the retailer and the coop corresponding to the coop's each pricing strategy. To avoid repetition, we shall provide the details for the case when the coop adopts pricing strategy 1 , and relegate the details for other cases to Online Appendix.

\footnotetext{
${ }^{11}$ For tractability, we assume that the consumption rate of each consumer is exogenously given and independent of price. This assumption is reasonable for a product that is not very price sensitive or a product with stable consumption rate. Examples include tooth paste, toilet paper, milk, bread, etc. We discuss this assumption in more detail in $\S 6$.
} 
Coop adopts pricing strategy 1 . When the coop sets $F \leq \delta l$ according to pricing strategy 1 , both high- and low-consumption consumers shopping at the coop become coop members by paying an upfront membership fee $F$ and a member-only price $(p-\delta)$ per unit. Any consumer shopping at the retailer has to pay $r$ per unit. Thus, high-consumption consumers shop at the coop if $F+(p-\delta) h-S \leq r \cdot h$, and low-consumption consumers shop at the coop if $F+(p-\delta) l-S \leq r \cdot l .^{12}$ Because $S \sim U[0, v]$, the proportion of type $j(\in\{h, l\})$ consumers who buy from the coop and the retailer can be written as $\min \{\max \{[v-(F+(p-\delta-r) \cdot j)] / v, 0\}, 1\}$ and $\min \{\max \{[F+(p-\delta-$ $r) \cdot j] / v, 0\}, 1\}$, respectively. Lemma EC.1 in Online Appendix shows that the coop can restrict attention to $(F, p, \delta)$ such that $v \geq F+(p-\delta-r) \cdot j \geq 0$ for all $j \in\{h, l\}$ without loss of optimality, because otherwise, the coop can weakly improve its objective by choosing $(F, p, \delta)$ that satisfies these conditions. Because the proportion of high-consumption type is $\alpha$, we can determine the sales quantities for each store when the coop adopts pricing strategy 1 as depicted in Table 2.

Table 2 Sales quantities at different stores when the coop adopts pricing strategy 1.

\begin{tabular}{c|c|c}
\hline & retailer & food coop \\
\hline high-consumption type & $\frac{\alpha}{v} \cdot[F+(p-\delta-r) \cdot h] \cdot h$ & $\underline{\alpha} \cdot[v-(F+(p-\delta-r) \cdot h)] \cdot h$ \\
low-consumption type & $\frac{1-\alpha}{v} \cdot[F+(p-\delta-r) \cdot l] \cdot l$ & $\frac{1-\alpha}{v} \cdot[v-(F+(p-\delta-r) \cdot l)] \cdot l$ \\
\hline
\end{tabular}

Let subscripts $r, c$, and 1 denote the retailer, the coop, and the coop's pricing strategy 1 (i.e., when the coop enters by setting $F \leq \delta l$ ), respectively. By considering the quantities in Table 2 along with the retailer's unit cost $w_{r}$ and the coop's unit cost $w$, we can drive the retailer's sales quantity $Q_{r 1}$, the coop's sales quantity $Q_{c 1}$, and the corresponding retailer profit $\Pi_{r 1}$ and coop profit $\Pi_{c 1}$, which incorporates the operating cost $k$ as:

$$
\begin{aligned}
Q_{r 1}= & \frac{\alpha}{v} \cdot(F+(p-\delta-r) \cdot h) \cdot h+\frac{1-\alpha}{v} \cdot(F+(p-\delta-r) \cdot l) \cdot l, \\
Q_{c 1}= & \frac{\alpha}{v} \cdot[v-(F+(p-\delta-r) \cdot h)] \cdot h+\frac{1-\alpha}{v} \cdot[v-(F+(p-\delta-r) \cdot l)] \cdot l, \\
\Pi_{r 1}= & \left(r-w_{r}\right) \cdot Q_{r 1}, \\
\Pi_{c 1}= & \frac{\alpha}{v} \cdot[v-(F+(p-\delta-r) \cdot h)] \cdot[F+(p-\delta-w) h] \\
& +\frac{1-\alpha}{v} \cdot[v-(F+(p-\delta-r) \cdot l)] \cdot[F+(p-\delta-w) l]-k .
\end{aligned}
$$

Coop adopts pricing strategy 2. When the coop sets $F \in[\delta l, \delta h]$ according to pricing strategy 2, only high-consumption consumers become members, and low-consumption consumers do not. Using the same approach as above, the retailer's sales quantity $Q_{r 2}$, the coop's sales quantity $Q_{c 2}$, and their corresponding profits $\Pi_{r 2}$ and $\Pi_{c 2}$ can be written as follows (we refer the reader to Online Appendix for details of how these quantities are derived):

$$
Q_{r 2}=\frac{\alpha}{v} \cdot(F+(p-\delta-r) \cdot h) \cdot h+\frac{1-\alpha}{v} \cdot(p-r) \cdot l \cdot l
$$

${ }^{12}$ We assume that each consumer shops either at the retailer or at the coop so that the market is fully covered. A fully-covered market is the conventional approach to ensure tractability (cf. Fudenberg and Tirole 1991, page 15). 


$$
\begin{aligned}
Q_{c 2}= & \frac{\alpha}{v} \cdot[v-(F+(p-\delta-r) \cdot h)] \cdot h+\frac{1-\alpha}{v} \cdot[v-(p-r) \cdot l] \cdot l, \\
\Pi_{r 2}= & \left(r-w_{r}\right) \cdot Q_{r 2}, \\
\Pi_{c 2}= & \frac{\alpha}{v} \cdot[v-(F+(p-\delta-r) \cdot h)] \cdot[F+(p-\delta-w) h] \\
& +\frac{1-\alpha}{v} \cdot[v-(p-r) \cdot l] \cdot(p-w) \cdot l-k .
\end{aligned}
$$

Coop adopts pricing strategy 3. When the coop sets $F \geq \delta h$ according to pricing strategy 3 , no coop shoppers will become members. Using the same approach as above, we obtain:

$$
\begin{aligned}
Q_{r 3} & =\frac{\alpha}{v} \cdot(p-r) \cdot h \cdot h+\frac{1-\alpha}{v} \cdot(p-r) \cdot l \cdot l, \\
Q_{c 3} & =\frac{\alpha}{v} \cdot[v-(p-r) \cdot h] \cdot h+\frac{1-\alpha}{v} \cdot[v-(p-r) \cdot l] \cdot l, \\
\Pi_{r 3} & =\left(r-w_{r}\right) \cdot Q_{r 3} \\
\Pi_{c 3} & =(p-w) \cdot Q_{c 3}-k
\end{aligned}
$$

\subsection{Coop's Social-Mission-Specific Problem}

As discussed in $\S 1$, most coops focus on either social mission (A) or (B). Under mission (A), the coop is committed to maximizing its reserve (made out of profit) as a means to support local community needs. Thus, by considering the pricing strategies in $§ 3.1$ along with the coop's profit in (4), (8), and (12), the coop with mission (A) (in short, coop A) solves the following problem:

$$
\Pi_{c}^{A}=\max \left\{\max _{F \leq \delta l, p, \delta}\left\{\Pi_{c 1}\right\}, \max _{F \in[\delta l, \delta h], p, \delta}\left\{\Pi_{c 2}\right\}, \max _{F \geq \delta h, p, \delta}\left\{\Pi_{c 3}\right\}\right\} .
$$

Here, we use superscript $A$ to denote the social mission of the coop.

Under social mission (B), the coop is committed to maximizing its sales to support the local economy (i.e., create more local jobs, sell more locally produced products, etc.). By considering the pricing strategies in $§ 3.1$ and the coop's sales quantities in (2), (6), and (10), coop B solves:

$$
Q_{c}^{B}=\max \left\{\max _{F \leq \delta l, p, \delta}\left\{Q_{c 1}\right\}, \max _{F \in[\delta l, \delta h], p, \delta}\left\{Q_{c 2}\right\}, \max _{F \geq \delta h, p, \delta}\left\{Q_{c 3}\right\}\right\} .
$$

\subsection{Coop's Entry and Pricing Strategy and Retailer's Deterrence Strategy}

So far, we have established the requisites that will enable us to examine our three research questions stated in $§ 1$. Specifically, we examine the coop's entry and pricing strategy and the retailer's decision of whether to deter the entry of the coop (in short, "deterrence strategy") via backward induction as depicted in Figure 1.

Coop's Entry Strategy. Given the retailer's price $r$ set in period 1, coop A decides whether to enter by determining its optimal profit $\Pi_{c}^{A}$ as given in (13). Coop A can afford to enter the market only if $\Pi_{c}^{A} \geq 0$. Similarly, coop B decides whether to enter the market by determining its corresponding profit $\Pi_{c}^{B}$ that is retrieved from the optimal sales quantity $Q_{c}^{B}$ as given in (14). Specifically, coop B can afford to enter the market if the corresponding profit $\Pi_{c}^{B}$ is non-negative. 
As a preview, we shall show that there exists some function of the retailer's price $r$ denoted by $\tau(r)$ such that, regardless of the underlying social mission, the coop can afford to enter the market only when its fixed annual operating cost $k \leq \tau(r)$.

Retailer's Deterrence Strategy. Anticipating that the coop's entry decision depends on whether or not $k \leq \tau(r)$, the retailer has two strategic choices: (1) "deter" the coop's entry by setting $r$ so that $k>\tau(r)$, or (2) "tolerate" the coop's entry by selecting the optimal retail price $r$ subject to the coop's entry condition $k \leq \tau(r)$. By comparing retailer profits under these two choices, the retailer can determine its optimal strategy of whether to deter or tolerate the coop's entry.

In the remainder of the paper, we examine the coop's entry and pricing strategy by characterizing $\tau(r)$ in $\S 4$, and then we examine the retailer's optimal deterrence strategy (deter or tolerate) in $\S 5$.

\section{Optimal Pricing Strategy for Coop A and Coop B}

We now characterize the optimal pricing strategy for coop A and coop B, and determine how the coop's entry affects the retailer.

\subsection{Coop A: Maximize Reserve to Support Local Community}

Because coop A's objective is to maximize its reserve generated from the profit to support local community needs, let us first determine coop A's maximum profit $\Pi_{c}^{A}$ upon entry. For any given retailer price $r$, observe from (13) that $\Pi_{c}^{A}$ can be obtained by comparing the optimal profits $\Pi_{c 1}^{A}, \Pi_{c 2}^{A}, \Pi_{c 3}^{A}$ associated with the three corresponding pricing strategies for coop A presented in $\S 3.2$, where $\Pi_{c 1}^{A}=\max _{F \leq \delta l, p, \delta}\left\{\Pi_{c 1}\right\}, \Pi_{c 2}^{A}=\max _{F \in[\delta l, \delta h], p, \delta}\left\{\Pi_{c 2}\right\}$, and $\Pi_{c 3}^{A}=\max _{F \geq \delta h, p, \delta}\left\{\Pi_{c 3}\right\}$, and $\Pi_{c 1}, \Pi_{c 2}$, and $\Pi_{c 3}$ are as in (4), (8), and (12), respectively. In preparation, we solve these three sub-problems.

As a preview, the optimal pricing decision of coop $\mathrm{A}$ is based on the social-benefit level $v$, so the corresponding sales quantities generated from high- and low-consumption consumers of coop A and the retailer also depend on $v$. Before we present coop A's optimal decisions, let us first present Proposition 1 that shows the impact of $v$ on the optimal sales quantities of coop A and the retailer. For ease of exposition, we use $Q_{i, j}^{A}$ to represent the sales quantity of each segment, where $i \in\{r, c\}$ ( $r$ and $c$ stand for the retailer and the coop, respectively) and $j \in\{h, l\}$ ( $h$ and $l$ stand for the highand low-consumption consumer types, respectively). All proofs are presented in Appendix.

Proposition 1. Suppose that coop A's annual operating cost $k$ is sufficiently small, and that for a given retailer price $r$, coop $A$ chooses the annual membership fee $F$, price $p$, and member-only discount $\delta$ that maximizes its profit. Then, the corresponding sales quantities of coop $A$ and the retailer $Q_{i, j}^{A}, i \in\{r, c\}$ and $j \in\{h, l\}$ satisfy the following:

(a) When $v>|r-w| \cdot h$, coop $A$ and the retailer can co-exist and share both high- and lowconsumption consumer markets (i.e., $Q_{i, j}^{A}>0, \forall i, j$ ). 
(b) When $v \in(|r-w| \cdot l,|r-w| \cdot h]$, coop $A$ and the retailer only share the low-consumption consumer market (i.e., $Q_{i, l}^{A} \geq 0 \forall i$ ). Furthermore, if $r \geq w$, then coop A captures the whole highconsumption consumer market (i.e., $Q_{c, h}^{A}=\alpha \cdot h$ and $Q_{r, h}^{A}=0$ ); if $r<w$, then the retailer captures the whole high-consumption consumer market (i.e, $Q_{c, h}^{A}=0$ and $Q_{r, h}^{A}=\alpha \cdot h$ ).

(c) When $v \leq|r-w| \cdot l$, then it is a monopoly market; specifically, if $r \geq w$, then coop A captures the whole market (i.e., $\left.Q_{c, h}^{A}=\alpha \cdot h, Q_{c, l}^{A}=(1-\alpha) \cdot l, Q_{r, h}^{A}=Q_{r, l}^{A}=0\right)$; if $r<w$, then the retailer captures the whole market (i.e., $Q_{c, h}^{A}=Q_{c, l}^{A}=0, Q_{r, h}^{A}=\alpha \cdot h, Q_{r, l}^{A}=(1-\alpha) \cdot l$ ).

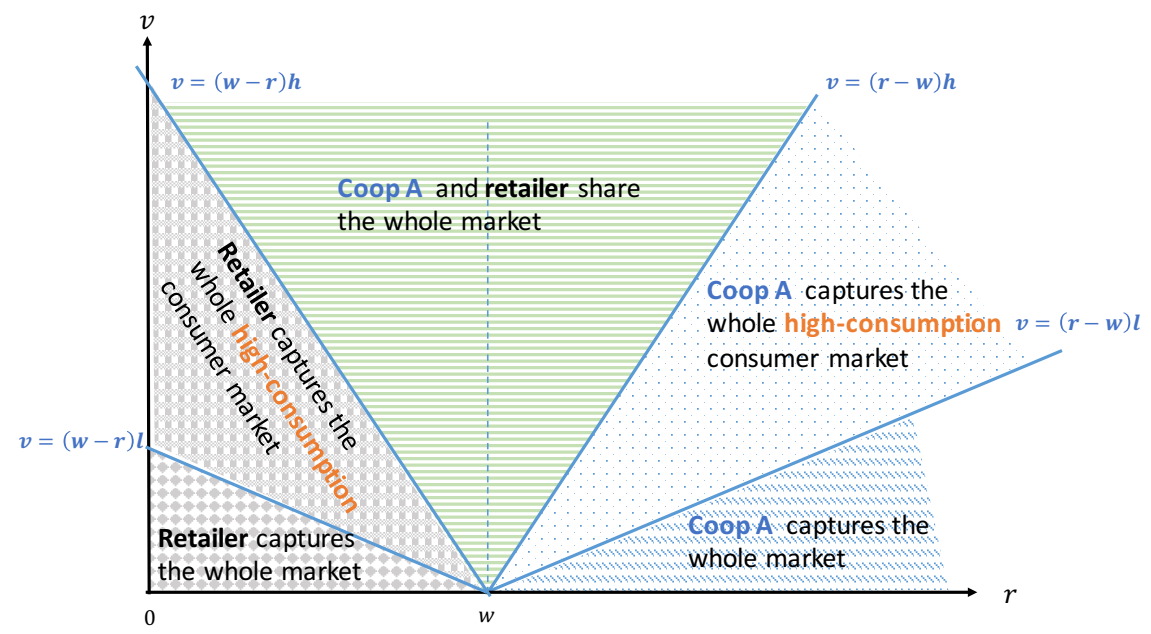

Figure 2 Market shares of coop A and the retailer under different values of the social-benefit level $v$ and the retailer's price $r$.

We use Figure 2 to illustrate the results in Proposition 1. Proposition 1(a) shows that when the social-benefit level $v$ is sufficiently large, coop $\mathrm{A}$ and the retailer share the whole market and make positive profits. When the retailer charges a price $r$ below the coop's unit cost $w$ (as in the left-hand side of Figure 2) and $v$ is small, the retailer captures all of the high-consumption consumer market and may even capture the whole market. Thus, a large $v$ can help coop A survive (under a sufficiently low fixed annual operating cost $k$ ). This is somewhat intuitive. Perhaps more interestingly, when $r \geq w$ (right-hand side of Figure 2), a large $v$ can also help the retailer survive. This is because as consumers have higher social-benefit level $v$, coop A can make a larger profit by charging a higher price $p$ and focusing on consumers with high social benefit, and leaving consumers with less social benefit to the retailer. Next, by considering the difference between the coop and the retailer's unit cost as $\Delta w$ (i.e., $w=w_{r}+\Delta w$, and $\Delta w$ can be either greater or smaller than 0 ) and given that the retailer will charge $r$ greater than its unit cost $w_{r}$, we can further derive the following Corollary 1 based on Proposition 1.

Corollary 1. Given retailer price $r \geq w_{r}$ and coop $A$ 's unit cost $w=w_{r}+\Delta w$, suppose that coop $A$ 's annual operating cost $k$ is sufficiently small so that coop A can enter the market. 
(a) Suppose $\Delta w \leq 0$ so that coop A's unit cost is lower than the retailer. Then, coop A can (1) capture the whole market if $v \leq-\Delta w \cdot l$; and (2) capture the whole high-consumption consumer market if $v \in(-\Delta w \cdot l,-\Delta w \cdot h]$.

(b) Suppose $\Delta w>0$ so that coop A's unit cost is higher than the retailer. Then, if the retailer charges a sufficiently low retail price $r$, coop A may not be able to (1) get any market share from one or both market if $v \leq \Delta w \cdot l$; and (2) get any high-consumption consumer market share if $v \in(\Delta w \cdot l, \Delta w \cdot h]$.

Corollary 1(a) implies that when the coop has a lower unit cost than the retailer, it is possible for the coop to drive the retailer out of one or both consumer market when the social benefit level $v$ is not sufficiently high. This is because if the coop's unit cost is very low, then only when the social benefit level $v$ is sufficiently high will coop A focus on consumers with high social benefit and leave consumers with less social benefit to the retailer. Corollary 1(b) reveals that when the coop's unit cost is higher than the retailer's, and the social benefit level $v$ is not sufficiently high, then it is still possible that the coop may not be able to share one or both consumer market with the retailer. This is because when the coop's unit price is too high and the social-benefit level is low, the retailer can capture all of the high-consumption consumer market and even the whole market by charging a price $r$ below the coop's unit cost $w$.

Proposition 1(b)-(c) shows that when $v$ is not sufficiently large, either the retailer or the coop captures one or both of the consumer markets. Because we are more interested in identifying the cases where the coop and the retailer can co-exist and compete, for the rest of $\S 4.1$, we shall focus on the case where $v>|r-w| \cdot h$ so that both coop A and the retailer share the whole market as shown in Figure 2. (For completeness, the optimal pricing decision of coop A in the case when $v \leq|r-w| \cdot h$ and the corresponding results are relegated to the proof of Proposition 1 in Appendix.) More formally, throughout $\S 4.1$, we impose the following assumption to ensure that coop A and the retailer can co-exist and compete for both high- and low-consumption consumer markets.

Assumption 1. The retailer's price $r$, the coop's unit cost $w$, high-consumption amount $h$, and social-benefit level $v$ satisfy $v \geq|r-w| \cdot h$.

So far, our analysis is based on a given coop's pricing strategies $(1,2$, or 3$)$. We now characterize coop A's optimal pricing strategy for each of the three pricing strategies presented in $\S 3.1$.

4.1.1. Coop A adopts pricing strategy 1. When coop A sets $F \leq \delta l$, both high- and lowconsumption coop shoppers will become members by paying an upfront membership fee $F$ and a member-only price $\tilde{p} \equiv(p-\delta)$ per unit. Hence, by substituting $\tilde{p}=(p-\delta)$ in $(4)$, and eliminating the decision $\delta$ along with the constraint $F \leq \delta l$, coop A's problem associated with strategy 1, i.e., $\Pi_{c 1}^{A}=\max _{F \leq \delta l, p, \delta}\left\{\Pi_{c 1}\right\}$, can be reformulated as follows: 


$$
\begin{aligned}
\max _{F, \tilde{p}} & \frac{\alpha}{v} \cdot[v-(F+(\tilde{p}-r) \cdot h)] \cdot(F+(\tilde{p}-w) \cdot h)+\frac{1-\alpha}{v} \cdot[v-(F+(\tilde{p}-r) \cdot l)] \cdot(F+(\tilde{p}-w) \cdot l)-k \\
\text { s.t. } & F+(\tilde{p}-r) \cdot l \geq 0, \\
& F+(\tilde{p}-r) \cdot h \geq 0, \\
& v-(F+(\tilde{p}-r) \cdot h) \geq 0, \\
& v-(F+(\tilde{p}-r) \cdot l) \geq 0, \\
& F \geq 0, \tilde{p} \geq 0 .
\end{aligned}
$$

Constraints (16)-(17) and (18)-(19) ensure that the solution of (15) yields non-negative market shares for the retailer and the coop, respectively. Although the above mathematical program only yields the optimal upfront membership fee $F_{1}^{A}$ and member-only price $\tilde{p}_{1}^{A}$, the coop can implement this optimal solution by setting any optimal member-only discount $\delta_{1}^{A} \geq F_{1}^{A} / l$ and a corresponding optimal price $p_{1}^{A}=\tilde{p}_{1}^{A}+\delta_{1}^{A}$. The following lemma characterizes coop A's optimal decisions.

Lemma 1. Suppose the retailer's price is $r$ and coop A adopts pricing strategy 1. Then it is optimal for coop $A$ to set $F_{1}^{A}=\frac{v}{2}, p_{1}^{A}=\frac{r+w}{2}+\delta_{1}^{A}$, and $\delta_{1}^{A} \geq \frac{v}{2 l}$ (so that $\left.F_{1}^{A} \leq \delta_{1}^{A} l\right)$. The corresponding optimal sales quantity $Q_{c 1}^{A}=\frac{1-\alpha}{2 v}(v+(r-w) \cdot l) \cdot l+\frac{\alpha}{2 v} \cdot(v+(r-w) \cdot h) \cdot h$ and profit $\Pi_{c 1}^{A}=\tau-k$, where

$$
\tau \equiv \frac{1}{4 v}\left[(1-\alpha)(v+l(r-w))^{2}+\alpha \cdot(v+h(r-w))^{2}\right] .
$$

Substituting the optimal $\left(F_{1}^{A}, p_{1}^{A}, \delta_{1}^{A}\right)$ stated in Lemma 1 into Table 2, we can retrieve the corresponding sales quantities at coop $\mathrm{A}$ and the retailer as summarized in Table 3. Lemma 1 has

Table 3 Sales quantities at different stores when coop A adopts pricing strategy 1.

\begin{tabular}{c|c|c}
\hline & retailer & food coop \\
\hline high-consumption type & $\frac{\alpha}{2 v}[v-h(r-w)] \cdot h$ & $\frac{\alpha}{2 v}[v+h(r-w)] \cdot h$ \\
low-consumption type & $\frac{1-\alpha}{2 v}[v-l(r-w)] \cdot l$ & $\frac{1-\alpha}{2 v}[v+l(r-w)] \cdot l$ \\
\hline
\end{tabular}

interesting implications about which pricing tools (price or membership fee) coop A should adjust when there is a change in factors that are exogenous to coop A. First, when consumers have a higher social-benefit level $v$, they are willing to pay more to shop at the coop, so coop A can afford to charge them more. Lemma 1 shows that coop A should charge a higher membership fee $F_{1}^{A}$, but not a higher member-only price $\tilde{p}^{A}$, because this strategy allows the coop to extract larger profits from both high-consumption and low-consumption consumers. Second, when the retailer's price $r$ is higher, coop A again can afford to charge consumers more, but this time, coop A should increase its member-only price $\tilde{p}^{A}$ rather than its fixed membership fee $F_{1}^{A}$. Third, when coop A's wholesale price $w$ is higher, coop A should reflect this increase to its member-only price $\tilde{p}^{A}$ but not its membership fee $F_{1}^{A}$. Lemma 1 shows that, although the specific pricing tool that the coop A should adjust differs depending on the exogenous factor, coop A benefits from a higher social-benefit level $v$, a higher retailer's price $r$, and a lower wholesale price $w$ by achieving a larger sales quantity $Q_{c 1}^{A}$ and profit $\Pi_{c 1}^{A}$. 
4.1.2. Coop A adopts pricing strategy 2. When coop A sets $F \in[\delta l, \delta h]$, only highconsumption consumers become members. The member-only discount $\delta$ per unit leads to annual cost savings of $\delta h$ for high consumption consumers, so the net membership fee they pay becomes $\tilde{F} \equiv F-\delta \cdot h \leq 0$. Because $F \in[\delta l, \delta h]$, we can infer that $\tilde{F}+\delta h \geq \delta l$, so $\delta \geq-\frac{\tilde{F}}{h-l}$. To ensure the member-only price $p-\delta \geq 0$, we require that $p \geq \delta \geq-\frac{\tilde{F}}{h-l}$. By substituting $\tilde{F}=F-\delta \cdot h$ into (8) and eliminating the decision $\delta$ along with the constraint $\delta \geq-\frac{\tilde{F}}{h-l}$, coop A's problem associated with strategy 2 can be reformulated as follows:

$$
\begin{aligned}
\max _{\tilde{F}, p} & \frac{\alpha}{v}[v-(\tilde{F}+(p-r) h)] \cdot(\tilde{F}+(p-w) h)+\frac{1-\alpha}{v}(v-(p-r) l) \cdot(p-w) \cdot l-k \\
\text { s.t. } & \tilde{F}+(p-r) \cdot h \geq 0, \\
& p-r \geq 0 \\
& v-(\tilde{F}+(p-r) \cdot h) \geq 0, \\
& v-(p-r) \cdot l \geq 0, \\
& \tilde{F} \leq 0, p \geq-\frac{\tilde{F}}{h-l} .
\end{aligned}
$$

As in the case of strategy 1, the constraints ensure that the solution to (22) yields non-negative market shares for the retailer and the coop. Although the above mathematical program only yields the optimal net membership fee $\tilde{F}_{2}^{A}$ and the optimal price $p_{2}^{A}$, the coop can implement this optimal solution by choosing any optimal member-only discount $\delta_{2}^{A} \geq-\frac{\tilde{F}_{2}^{A}}{h-l}$ and a corresponding optimal membership fee $F_{2}^{A}=\tilde{F}_{2}^{A}+\delta_{2}^{A} h$.

Lemma 2. Suppose the retailer's price is $r$ and coop A adopts pricing strategy 2. Then coop A's optimal decisions are: $F_{2}^{A}=\delta_{2}^{A} \cdot h-\frac{(h-l) v}{2 l}, p_{2}^{A}=\frac{v+l(r+w)}{2 l}$, and $\delta_{2}^{A} \in\left[\frac{v}{2 l}, \frac{v}{2 l}+\frac{r-w}{2}\right]$ (so that $F_{2}^{A} \in\left[\delta_{2}^{A} l, \delta_{2}^{A} h\right]$ ). The corresponding optimal sales quantity $Q_{c 2}^{A}=\frac{1-\alpha}{2 v}(v+(r-w) \cdot l) \cdot l+\frac{\alpha}{2 v} \cdot(v+(r-w) \cdot h) \cdot h$ and profit $\Pi_{c 2}^{A}=\tau-k$, where $\tau$ is given in (21).

Different from strategy 1, Lemma 2 implies that when coop A chooses to adopt strategy 2, it induces only high-consumption consumers to become members. An interesting observation from Lemmas 1 and 2 is that the optimal profits and sales quantities are equal (i.e., $\Pi_{c 1}^{A}=\Pi_{c 2}^{A} \equiv \tau-k$ and $Q_{c 1}^{A}=Q_{c 2}^{A}$ ) under strategies 1 and 2. This is because regardless of whether coop A adopts strategy 1 or 2, each high- consumption coop shopper pays $F_{1}^{A}+\left(p_{1}^{A}-\delta_{1}^{A}\right) \cdot h=F_{2}^{A}+\left(p_{2}^{A}-\delta_{2}^{A}\right) \cdot h=\frac{v}{2}+\frac{(r+w) h}{2}$ and each low-consumption coop shopper pays $F_{1}^{A}+\left(p_{1}^{A}-\delta_{1}^{A}\right) \cdot l=p_{2}^{A} \cdot l=\frac{v}{2}+\frac{(r+w) l}{2}$ annually. Therefore, from the perspective of sales quantity and profit, coop $\mathrm{A}$ is indifferent between strategies 1 and $2 .{ }^{13}$ Yet, it is worth noting that the coop's pricing decisions are more complex and the impact of exogenous factors on coop A's optimal pricing decisions is more nuanced under strategy 2 as compared to strategy 1. For instance, a larger social-benefit level $v$ may lead to larger price for the coop, unlike as in strategy 1 where the price does not change with $v$.

${ }^{13}$ As an interesting observation, if we set $\delta_{1}^{A}=\delta_{2}^{A}=\frac{v}{2 l}$ as the boundary case, the corresponding $\left(F_{1}^{A}, p_{1}^{A}\right)=\left(F_{2}^{A}, p_{2}^{A}\right)=$ $\left(\frac{v}{2}, \frac{v+l(r+w)}{2 l}\right)$ so that $F_{1}^{A}=\delta_{1}^{A} \cdot l=F_{2}^{A}=\delta_{2}^{A} l$. 
4.1.3. Coop A adopts pricing strategy 3. When coop A sets $F \geq \delta h$, no consumers will become members, so $(F, \delta)$ are no longer relevant decisions. By solving the optimal price $p_{3}^{A}$ under strategy 3 and comparing the corresponding profit $\Pi_{c 3}^{A}$ with $\tau-k$, we obtain that $\Pi_{c 3}^{A} \leq \tau-k$. Thus, coop A will never choose pricing strategy 3 because it can obtain a larger profit under other strategies. Therefore, we omit the analysis of pricing strategy 3 here, while referring the reader to Online Appendix for details.

Lemma 3. Suppose that the retailer price is $r$ and coop A adopts pricing strategy 3. Then the optimal profit $\Pi_{c 3}^{A} \leq \tau-k$ so that strategy 3 is dominated by strategy 1 and 2.

4.1.4. Coop A's optimal pricing strategy and entry condition. By examining the optimal profits as reported in Lemmas 1, 2, and 3, we can conclude that coop A's optimal profit $\Pi_{c}^{A}$ defined in (13) satisfies $\Pi_{c}^{A}=\tau-k$, where $\tau$ is given in (21). In view of the fact that coop A can only afford to enter the market when $\Pi_{c}^{A}=\tau-k \geq 0$, we obtain the following proposition.

Proposition 2. Suppose that the retailer's price is $r$ and coop A's fixed annual operating cost is $k$. Then coop A can afford to enter the market only when $\tau \geq k$, where $\tau$ is given in (21). In the event when coop $A$ enters the market, it is optimal for coop A to deploy strategy 1 as stated in Lemma 1 (or deploy strategy 2 as stated in Lemma 2) and generate a profit $\Pi_{c}^{A}=\tau-k$.

Proposition 2 implies that a membership system (i.e., setting membership fee $F$ and member discount $\delta$ ) helps coop A earn a larger profit because it entices more people to shop at the coop, and hence leads to a larger sales quantity. Moreover, Proposition 2 reveals that it is important for the coop to induce high-consumption consumers to become members; while it does not matter whether the low-consumption consumers become members or not. This is because under the coop's optimal pricing strategy, a low-consumption consumer incurs the same cost when shopping at the coop, regardless of whether she becomes a member or not.

\subsection{Coop B: Maximum Sales for Supporting Local Economy}

We next analyze the optimal pricing strategy of coop B, which aims to maximize its total sales quantities. Recall from Proposition 1 that when coop A intends to maximize its profit (as a means to generate reserve to support local needs), a large social-benefit level $v$ can also help the retailer survive. However, different from coop A, coop B intends to maximize total sales quantity to support the local economy, so a large $v$ cannot ensure the co-existence of coop B and the retailer. Specifically, when the coop B's fixed annual operating cost $k \leq(\alpha h+(1-\alpha) l) \cdot(r-w)$, it is always optimal for coop B to set $F=0, \delta=0$ and $p=r$ so as to capture the entire market (i.e., $Q_{c}^{B}=\alpha h+(1-\alpha) l$ ) by also ensuring the corresponding profit $\Pi_{c}^{B}=(\alpha h+(1-\alpha) l) \cdot(r-w)-k \geq 0$. Only when $k>(\alpha h+(1-\alpha) l) \cdot(r-w)$, the retailer can have some positive market share.

Recall from Proposition 2 that when social-benefit level $v>|r-w| \cdot h$, the maximum profit that the coop can make is $\tau-k$, where $\tau$ is as given in (21). Therefore, in order for coop B to co-exist with the retailer by sharing both high- and low-consumption consumer markets, its fixed annual operating cost $k$ should take on moderate values (i.e., $k \in[\tilde{\tau}, \tau]$ as stated in Proposition 4 below). This is because when $k$ is too high, coop B cannot enter the market and when $k$ is too small, the coop will drive the retailer out of the market. 
Besides, when $k$ is very small, given the objective function to maximize the sales quantity, it is also possible for the coop B to charge a zero member-only price (i.e., $p-\delta=0$ ) and use membership fee to compensate for the loss. Yet, such practice violates antitrust regulations. The following proposition formally states the precise conditions under which coop B co-exists with the retailer by sharing both high- and low-consumption consumer markets, and charges a positive member-only price.

Proposition 3. Coop B co-exists with the retailer, and competes for both high-and low-consumption consumer markets with a positive member-only price (i.e., $p-\delta>0$ ) when the social benefit level $v \geq|r-w| \cdot h$ and the coop's annual operating cost $k \in(\tilde{\tau}, \tau]$, where $\tilde{\tau}=\max \left\{\tau-\frac{(r+w)^{2}\left(l^{2}(1-\alpha)+h^{2} \alpha\right)}{4 v}, \tau-\frac{\left(\frac{v}{h}+w-r\right)^{2}\left(l^{2}(1-\alpha)+h^{2} \alpha\right)}{4 v}\right\}$, and $\tau$ is given in (21).

As we are more interested in the more practically-relevant case where the coop sets a positive member-only price and can co-exist with the retailer, we impose the following Assumption 2 throughout $\S 4.2$ when we discuss the pricing strategy of coop B.

ASSUMPTION 2. The retailer's price $r$, the coop's unit cost $w$, high-consumption amount $h$, and social-benefit level $v$ satisfy $v \geq|r-w| \cdot h$; and the coop's annual operating cost $k \in(\tilde{\tau}, \tau]$.

Knowing that coop B maximizes its sales quantity, let us first determine coop B's maximum sales quantity $Q_{c}^{B}$ upon entry. Observe from (14) that the maximum sales quantity $Q_{c}^{B}$ can be obtained by comparing the maximum sales quantities $Q_{c 1}^{B}, Q_{c 2}^{B}, Q_{c 3}^{B}$ associated with three different pricing strategies for coop B as presented in $\S 3.3$, where $Q_{c 1}^{B}=\max _{F \leq \delta l, p, \delta}\left\{Q_{c 1}\right\}, Q_{c 2}^{B}=\max _{F \in[\delta l, \delta h], p, \delta}\left\{Q_{c 2}\right\}$, and $Q_{c 3}^{B}=\max _{F \geq \delta h, p, \delta}\left\{Q_{c 3}\right\}$, and $Q_{c 1}, Q_{c 2}$, and $Q_{c 3}$ are given in (2), (6), and (10); respectively. We first solve these three sub-problems.

4.2.1. Coop B adopts pricing strategy 1. When coop B sets $F \leq \delta l$, both high- and low-consumption coop shoppers will become members by paying membership fee $F$ and a member-only price $\tilde{p}=(p-\delta)$ per unit. Coop B's problem associated with strategy 1 is to maximize its total sales quantity in (2) subject to making non-negative profit; i.e., $\Pi_{c 1}(F, p, \delta) \geq 0$ (where $\Pi_{c 1}(F, p, \delta)$ is given in (4)). Specifically, by using the same approach as presented in $\S 4.1 .1$, we reformulate coop B's problem as:

$$
\begin{aligned}
Q_{c 1}^{B}=\max _{F, \tilde{p}} & \frac{\alpha}{v} \cdot[v-(F+(\tilde{p}-r) \cdot h)] \cdot h+\frac{1-\alpha}{v} \cdot[v-(F+(\tilde{p}-r) \cdot l)] \cdot l, \\
\text { s.t. } & (16),(17),(18),(19),(20), \\
& \Pi_{c 1}=\frac{\alpha}{v} \cdot[v-(F+(\tilde{p}-r) \cdot h)] \cdot(F+(\tilde{p}-w) \cdot h)+\frac{1-\alpha}{v} \cdot[v-(F+(\tilde{p}-r) \cdot l)] \cdot(F+(\tilde{p}-w) \cdot l)-k \geq 0 .
\end{aligned}
$$

Lemma 4. Suppose the retailer's price is $r$ and coop $B$ adopts pricing strategy 1 . It is optimal for coop $B$ to set $F_{1}^{B}=\frac{v}{2}, p_{1}^{B}=\frac{r+w}{2}+\delta_{1}^{B}-\Delta^{*}$ and $\delta_{1}^{B} \geq \frac{v}{2 l}$ (so that $F_{1}^{B} \leq \delta_{1}^{B} l$ ), where

$$
\Delta^{*} \equiv \sqrt{\frac{v(\tau-k)}{l^{2}(1-\alpha)+h^{2} \alpha}} .
$$

Furthermore, coop B's maximum sales quantity $Q_{c 1}^{B}=\frac{\alpha}{2 v}\left[v+h\left(r-w+2 \Delta^{*}\right)\right] \cdot h+\frac{1-\alpha}{2 v} \cdot\left[v+l\left(r-w+2 \Delta^{*}\right)\right] \cdot l$ and its corresponding profit $\Pi_{c 1}^{B}=0$. 
Table 4 Sales quantity at different stores when coop B adopts pricing strategy 1.

\begin{tabular}{c|c|c}
\hline & retailer & food coop \\
\hline high-consumption type & $\frac{\alpha}{2 v}\left[v-h\left(r-w+2 \Delta^{*}\right)\right] \cdot h$ & $\frac{\alpha}{2 v}\left[v+h\left(r-w+2 \Delta^{*}\right)\right] \cdot h$ \\
low-consumption type & $\frac{1-\alpha}{2 v}\left[v-l\left(r-w+2 \Delta^{*}\right)\right] \cdot l$ & $\frac{1-\alpha}{2 v}\left[v+l\left(r-w+2 \Delta^{*}\right)\right] \cdot l$ \\
\hline
\end{tabular}

By substituting the optimal decisions as stated in Lemma 4 into the sales quantities given in Table 2, we can retrieve the corresponding sales quantities at different stores as summarized in Table 4.

By comparing the optimal decisions of coop A and coop B as stated in Lemmas 1 and 4, we can observe that coop B charges the same membership fee as coop A (i.e., $F_{1}^{B}=F_{1}^{A}$ ) and yet a lower member-only price (i.e., $\left.\left(p_{1}^{A}-\delta_{1}^{A}\right)-\left(p_{1}^{B}-\delta_{1}^{B}\right)=\Delta^{*} \geq 0\right)$. As a result, coop B achieves a higher sales quantity than coop A. If we analyze the member-only-price difference $\Delta^{*}$ between coop $\mathrm{A}$ and $\mathrm{B}$, we can observe that $\Delta^{*}$ increases with the social-benefit level $v$, and decreases with the coop's fixed annual operating cost $k$. As $k$ approaches $\tau$ in (21), $\Delta^{*}$ approaches zero, so the optimal member-only price of coop B converges to that of coop A.

4.2.2. Coop B adopts pricing strategy 2. When coop B sets $F \in[\delta l, \delta h]$, only high-consumption coop shoppers will become members. By defining $\tilde{F}=F-\delta h$ and using the same approach as explained in $\S 4.1 .2$, we can reformulate coop B's problem as:

$$
\begin{aligned}
Q_{c 2}^{B}=\max _{\tilde{F}, p} & \frac{\alpha}{v}[v-(\tilde{F}+(p-r) h)] \cdot h+\frac{1-\alpha}{v}(v-(p-r) l) \cdot l, \\
\text { s.t. } & (23),(24),(25),(26),(27), \\
& \Pi_{c 2}=\frac{\alpha}{v}[v-(\tilde{F}+(p-r) h)] \cdot(\tilde{F}+(p-w) h)+\frac{1-\alpha}{v}(v-(p-r) l) \cdot(p-w) \cdot l-k \geq 0 .
\end{aligned}
$$

Lemma 5. Suppose the retailer's price is $r$ and coop B adopts pricing strategy 2. Then coop B's optimal decisions are: $F_{2}^{B}=\delta_{2}^{B} \cdot h-\frac{(h-l) v}{2 l}, p_{2}^{B}=\frac{v+l(r+w)}{2 l}-\Delta^{*}$ and $\delta_{2}^{B} \in\left[\frac{v}{2 l}, \frac{v}{2 l}+\frac{r-w}{2}-\Delta^{*}\right]$, where $\Delta^{*}$ is given in (29). Also, coop B's maximum sales quantity $Q_{c 2}^{B}=\frac{\alpha}{2 v}\left[v+h\left(r-w+2 \Delta^{*}\right)\right] \cdot h+\frac{1-\alpha}{2 v} \cdot\left[v+l\left(r-w+2 \Delta^{*}\right)\right] \cdot l$, and the corresponding profit $\Pi_{c 2}^{B}=0$.

By considering Lemmas 2 and 5, let us compare the total cost of shopping for high- and low-consumption shoppers at coop A and B. Under strategy 2, each high-consumption shopper is a member, and the total shopping cost of a high-consumption shopper is $F_{2}^{A}+\left(p_{2}^{A}-\delta_{2}^{A}\right) h$ at coop A and $F_{2}^{B}+\left(p_{2}^{B}-\delta_{2}^{B}\right) h$ at coop B. From Lemmas 2 and 5 , it is easy to check that the total shopping cost is lower at coop B by the amount of $\Delta^{*} h$. Similarly, it is easy to check that each low-consumption shopper is a non-member, and the total shopping cost at coop B is lower than coop A by the amount of $\Delta * l$. As the total cost of shopping is lower at coop B, coop B can capture a higher market share in both segments and obtain a higher sales quantity. Also, by considering the definition of $\Delta^{*}$ given in (29), it is easy to check that coop B converges to coop A in terms of price and sales quantities as the coop's fixed annual operating cost $k$ approaches $\tau$ in (21).

4.2.3. Coop B adopts pricing strategy 3. When coop B sets $F \geq \delta h$, no coop shoppers will become members, so $(F, \delta)$ are no longer relevant decisions. Recall from Lemma 3 that, under strategy 3, coop A's profit $\Pi_{c 3}^{A} \leq \tau-k$ when it aims to maximize its profit. Hence, for coop B that aims to maximize its sales quantity, $\Pi_{c 3}^{B} \leq \Pi_{c 3}^{A} \leq \tau-k$. Also, by solving the optimal $p_{3}^{B}$ under strategy 3 and comparing the corresponding optimal sales quantity with those under strategies 1 and 2 as stated in Lemmas 4 and 5 , we 
obtain $Q_{c 3}^{B} \leq Q_{c 1}^{B}=Q_{c 2}^{B}$. Therefore, the following lemma shows that strategy 3 is dominated by strategy 1 and 2. (We refer the reader to Online Appendix for the analysis of coop B's pricing strategy 3.)

Lemma 6. Suppose the retailer's price is $r$ and coop B adopts strategy 3. Then the optimal sales quantity $Q_{c 3}^{B} \leq Q_{c 1}^{B}=Q_{c 2}^{B}$ so that strategy 3 is dominated by strategies 1 and 2.

4.2.4. Coop B's optimal strategy. The following proposition follows from Lemmas 4, 5, and 6 .

Proposition 4. It is optimal for coop $B$ to deploy strategy 1 as in Lemma 4 (or deploy strategy 2 as in lemma 5) to break even (i.e., $\Pi_{c 1}^{B}=\Pi_{c 2}^{B}=0$ ) and generate the maximum sales quantity $Q_{c}^{B}=Q_{c 1}^{B}=Q_{c 2}^{B}=$ $\frac{\alpha}{2 v}\left[v+h\left(r-w+2 \Delta^{*}\right)\right] \cdot h+\frac{1-\alpha}{2 v} \cdot\left[v+l\left(r-w+2 \Delta^{*}\right)\right] \cdot l$, where $\Delta^{*}$ is given in (29).

Proposition 4 shows that coop B can benefit from the membership system (i.e., setting both membership fee $F$ and member-only discount $\delta$ ) by achieving a higher sales quantity. Furthermore, coop B is indifferent between strategy 1 and 2 because high-consumption consumers will become members under both strategies; while each low-consumption coop shopper actually pays the same amount of money, regardless of whether she becomes a member or not.

\subsection{Impact of Coop's Entry on Retailer}

Recall from Propositions 2 and 4 that regardless of whether the coop's social mission is (A) or (B), it is optimal for the coop to adopt pricing strategy 1 (or strategy 2). By considering the pricing strategy 1 (the result is the same if we consider pricing strategy 2), we can apply Lemmas 1 and 4 to examine the impact of coop A's (or coop B's) entry on the retailer's sales quantity and profit.

To establish a benchmark, observe that, before the coop's entry, the retailer operates as a monopoly who captures the entire market so that its sales quantity is $Q_{r 0}=\alpha h+(1-\alpha) l$. Also, for any given retailer's price $r$ and unit cost $w_{r}$, the retailer earning is $\Pi_{r 0}=\left(r-w_{r}\right)(\alpha h+(1-\alpha) l)$.

By focusing on the case when Assumption 1 (i.e., $v \geq|r-w| \cdot h$ ) holds and coop A enters the market by deploying strategy 1 , we can use the sales quantities in Table 3 in $\S 4.1 .1$ to determine the retailer's sales quantity $Q_{r}^{A}$ so that the retailer's profit $\Pi_{r}^{A}=\left(r-w_{r}\right) Q_{r}^{A}$. Similarly, by focusing on the case when Assumption 2 (i.e., $v \geq|r-w| \cdot h$ and $k \in(\tilde{\tau}, \tau]$ ) holds and coop B adopts strategy 1, we can use the sales quantities presented in Table 4 in $\S 4.2 .1$ to determine the retailer's sales quantity $Q_{r}^{B}$ so that the retailer's profit $\Pi_{r}^{B}=\left(r-w_{r}\right) Q_{r}^{B}$. Specifically, retailer's sales quantities are:

$$
\begin{aligned}
& Q_{r}^{A}=\frac{\alpha}{2 v} \cdot[v-(r-w) h] \cdot h+\frac{1-\alpha}{2 v} \cdot[v-(r-w) l] \cdot l, \\
& Q_{r}^{B}=\frac{\alpha}{2 v} \cdot\left[v-\left(r-w+2 \Delta^{*}\right) h\right] \cdot h+\frac{1-\alpha}{2 v} \cdot\left[v-\left(r-w+2 \Delta^{*}\right) l\right] \cdot l .
\end{aligned}
$$

By comparing $Q_{r}^{A}$ with $Q_{r}^{B}$, and $\Pi_{r}^{A}=\left(r-w_{r}\right) Q_{r}^{A}$ with $\Pi_{r}^{B}=\left(r-w_{r}\right) Q_{r}^{B}$, we obtain the following corollary.

Corollary 2. Suppose the retailer's price is $r$, social-benefit level $v \geq|r-w| \cdot h$, and the coop's fixed annual operating cost $k \leq \tau$. Then the impact of a coop's entry is as follows:

1. Impact of Coop A's Entry: Under Assumption 1, upon coop A's entry, the retailer's sales quantity decreases from $Q_{r 0}$ to $Q_{r}^{A}$ (given in (31)) and the retailer's profit decreases from $\Pi_{r 0}$ to $\Pi_{r}^{A}$. 
2. Impact of Coop B's Entry: Under Assumption 2, upon coop B's entry, the retailer's sales quantity decreases from $Q_{r 0}$ to $Q_{r}^{B}$ (given in (32)) and the retailer's profit decreases from $\Pi_{r 0}$ to $\Pi_{r}^{B}$.

3. Relative Impact: The entry of coop B is more detrimental than the entry of coop A both in terms of lower sales quantity and profit: $\left(Q_{r 0}-Q_{r}^{B}\right) \geq\left(Q_{r 0}-Q_{r}^{A}\right)$ and $\left(\Pi_{r 0}-\Pi_{r}^{B}\right) \geq\left(\Pi_{r 0}-\Pi_{r}^{A}\right)$.

Corollary 2 reveals that the entry of coop B is more detrimental to a retailer than the entry of coop A. This is somewhat intuitive because coop B maximizes its sales quantity as long as it breaks even (as stated in Proposition 4), so it captures a higher sales quantity than coop A, which aims to maximize profit to support local community needs. Consequently, even though the retailer can co-exist and share both highand low-consumer markets with both coop A and B, the retailer suffers a bigger loss upon coop B's entry. In view of the damages caused by the coop's entry, should the retailer set its price $r$ strategically in period 1 (as shown in Figure 1) to deter the coop's entry? We address this question next.

\section{Retailer's Deterrence Strategy: Deter or Tolerate Coop's Entry}

Corollary 2 revealed that the coop's entry will encroach on retailer's market share and its profit. Yet, the retailer can anticipate that the coop (regardless of its social mission) can afford to enter the market only when its maximum profit $\Pi_{c}^{A}(r) \geq 0$. Thus, the retailer can deter the coop's entry by setting a sufficiently low price $r$ so that $\Pi_{c}^{A}(r)<0$, or can tolerate coop's entry by setting a higher price to avoid suffering from a low profit margin. In this section, we examine the retailer's deterrence strategy: whether it should deter or tolerate the coop's entry. Although we take retailer's price $r$ as given and impose Assumptions 1 and 2 in $\S 4$ for ease of exposition, we shall relax these assumptions when discussing the retailer's pricing and deterrence strategies.

\subsection{Deterrence Strategy of Coop A's Potential Entry}

We first examine the price that the retailer needs to charge in order to deter the coop from entering the market. Note that regardless of whether the coop's social mission is (A) or (B), the retailer can deter the coop by setting a price $r$ so that the maximum profit that the coop can make is below zero. Since coop A aims to maximize profit (to generate reserve), coop A's profit $\Pi_{c}^{A}(r)$ under its optimal pricing strategy is the maximum profit that coop A or B can make. We are interested in the case when the coop has some potential to enter and share both of the high- and low-consumption consumer market with the retailer at least for reasonable values of the retailer's price $r$, so we shall focus on the case when the coop's unit cost $w$, the retailer's unit cost $w_{r}$, and social-benefit level $v$ satisfy the condition $w_{r} \in\left[w-\frac{v}{h}, w+\frac{v}{h}\right]$ (i.e., $\left.\Delta w \in\left[-\frac{v}{h}, \frac{v}{h}\right]\right)$. This condition, which can be written as $v \geq\left|w-w_{r}\right| \cdot h=|\Delta w| \cdot h$, is analogous to Assumption 1 in $\S 4$, and it enables the coop and the retailer to coexist.

For any given $r$, by considering the optimal pricing strategy of coop A along with Proposition 1 for different ranges of the retailer's price $r$ as discussed in $\S 4.1$, we can derive the maximum profit that coop A or B can make, $\Pi_{c}^{A}(r)$, as follows:

$$
\Pi_{c}^{A}(r)= \begin{cases}\tau(r)-k=\frac{1}{4 v} \cdot\left[(1-\alpha)(v+l(r-w))^{2}+\alpha(v+h(r-w))^{2}\right]-k & w_{r} \leq r \leq \frac{v}{h}+w \\ \tau(r)-\frac{\alpha(v-h(r-w))^{2}}{4 v}-k & \frac{v}{h}+w \leq r \leq \frac{v}{l}+w . \\ \tau(r)-\frac{\alpha(v-h(r-w))^{2}}{4 v}-\frac{(1-\alpha)(v-l(r-w))^{2}}{4 v}-k & r \geq \frac{v}{l}+w\end{cases}
$$


Observe from (33) that the coop's maximum profit $\Pi_{c}^{A}(r)$ is increasing in the retailer's price $r$. When the retailer deters the coop's entry by setting its price $r\left(\geq w_{r}\right)$ so that the coop's profit $\Pi_{c}^{A}(r)<0$, the retailer captures the entire market with sales quantity $Q_{r 0}=\alpha h+(1-\alpha) l$, and obtains profit $\Pi_{r 0}=\left(r-w_{r}\right) \cdot(\alpha h+$ $(1-\alpha) l)$. Observe that the retailer's profit is increasing in $r$, so it is optimal for the retailer to set $r$ as large as possible subject to satisfying the deterrence condition $\Pi_{c}^{A}(r)<0$. Because $\Pi_{r 0}$ is continuous, the following problem gives a tight upper bound on the retailer's profit when deterring the coop:

$$
\Pi_{r}^{D}=\sup _{r \geq w_{r}}\left\{\Pi_{r 0} \mid \Pi_{c}^{A}(r)<0\right\} .
$$

This problem is solved by a retail price $r^{D}$ such that $\Pi_{c}^{A}\left(r^{D}\right)=0$. (Note that we use superscript " $D$ " to denote values corresponding to "deterrence" strategy.) By charging a slightly lower price than $r^{D}$, the retailer can deter the coop, and obtain a profit slightly below $\Pi_{r}^{D}$. We refer to $r^{D}$ as the "deterrence" price, and characterize it in the following proposition.

Proposition 5. Let $k_{0} \equiv \Pi_{c}^{A}\left(w_{r}\right)+k=\tau\left(w_{r}\right), k_{1} \equiv \Pi_{c}^{A}\left(\frac{v}{h}+w\right)+k=\frac{v\left[(h+l)^{2}+(h-l)(3 h+l) \alpha\right]}{4 h^{2}}$, and $k_{2} \equiv \Pi_{c}^{A}\left(\frac{v}{l}+\right.$ $w)+k=v\left(1+\alpha\left(\frac{h}{l}-1\right)\right)$, where $k_{0}<k_{1}<k_{2}$.

(a) When the coop's fixed annual operating cost $k \leq k_{0}$, the retailer cannot afford to deter the coop's entry;

(b) When $k>k_{0}$, the retailer can deter the coop's entry by setting its deterrence price at $r^{D}-\epsilon$, where $\epsilon \rightarrow 0^{+}$,

$$
\begin{aligned}
\lim _{\epsilon \rightarrow 0^{+}} \Pi_{c}^{A}\left(r^{D}-\epsilon\right) & =\Pi_{c}^{A}\left(r^{D}\right)=0, \text { and } \\
r^{D} & = \begin{cases}w+\frac{\sqrt{4 k v\left(l^{2}(1-\alpha)+h^{2} \alpha\right)-\alpha(1-\alpha) v^{2}(h-l)^{2}}-v(l(1-\alpha)+h \alpha)}{l^{2}(1-\alpha)+h^{2} \alpha} & k \in\left(k_{0}, k_{1}\right] \\
w+\frac{2 \sqrt{k l^{2} v(1-\alpha)+h v^{2} \alpha(l(1-\alpha)+h \alpha)}-v(2 h \alpha+l(1-\alpha))}{l^{2}(1-\alpha)} & k \in\left[k_{1}, k_{2}\right] \\
w+\frac{k}{l(1-\alpha)+h \alpha} & k \geq k_{2}\end{cases}
\end{aligned}
$$

Furthermore, the profit $\Pi_{r}^{D}$ in (34) can be characterized as $\Pi_{r}^{D}=\left(r^{D}-w_{r}\right) \cdot(\alpha h+(1-\alpha) l)$.

Proposition 5(a) shows that when the coop's fixed annual operating cost $k \leq k_{0}=\tau\left(w_{r}\right)$, the retailer cannot afford to deter the coop's entry. This is intuitive because when $k$ is sufficiently small, the minimum price the retailer can charge (i.e., the retailer's unit cost $w_{r}$ ) still does not deter the coop's entry. Proposition $5(\mathrm{~b})$ shows that the deterrence price $r^{D}$ is a piecewise function of $k$, because $r^{D}$ satisfies $\Pi_{c}^{A}\left(r^{D}\right)=0$, and $\Pi_{c}^{A}\left(r^{D}\right)$ is a piecewise function as shown in (33). By replacing $w$ with $w_{r}+\Delta w$ in the expression of $r^{D}$ as given by (35), we can further show that the deterrence price $r^{D}$ and the upper bound on the retailer's profit $\Pi_{r}^{D}$ in (34) satisfy the following property.

Corollary 3. $r^{D}$ and $\Pi_{r}^{D}$ characterized in Proposition 5 are both increasing in $\Delta w$ and $k$.

Corollary 3 shows that when the cost difference between the coop and the retailer $\Delta w$ increases, the retailer can afford to charge a higher deterrence price so as to achieve a higher profit because the coop's unit cost $w$ becomes higher. Corollary 3 also shows that when the coop's fixed annual operating cost $k$ increases, the retailer can afford to charge a higher price to deter the entry of the coop, so the retailer can achieve a higher profit. This result will come in handy when we discuss the retailer's deterrence strategy next. 


\subsection{Tolerance Strategy of Coop A's Potential Entry}

In this section, we characterize the retailer's deterrence strategy specifically for coop A. Proposition 5 reveals that, when $k>k_{0}$, the retailer can deter coop A's entry by setting a price slightly below the deterrence price $r^{D}$. Alternatively, the retailer can choose to tolerate coop A's entry by setting a higher price $r$ that satisfies $\Pi_{c}^{A}(r) \geq 0$. We next determine the optimal price $r^{A}$ that the retailer should set when it chooses to tolerate A's entry. (Note that we use superscript " $A$ " in the notations of retailer's price and profit when the retailer chooses to tolerate coop A.) To begin, we can determine the retailer's profit $\Pi_{r}^{A}(r)$ by considering the coop A's optimal pricing strategy for different ranges of $r$ as discussed in $§ 4.1$ as follows:

$$
\Pi_{r}^{A}(r)= \begin{cases}\left(r-w_{r}\right) \cdot\left[\frac{h \alpha}{2 v}(v-h(r-w))+\frac{l(1-\alpha)}{2 v}(v-l(r-w))\right] & w_{r} \leq r \leq \frac{v}{h}+w \\ \left(r-w_{r}\right) \cdot \frac{l(1-\alpha)}{2 v}(v-l(r-w)) & \frac{v}{h}+w \leq r \leq \frac{v}{l}+w . \\ 0 & r \geq \frac{v}{l}+w\end{cases}
$$

As such, when the retailer chooses to "tolerate" coop A's entry; i.e., when $\Pi_{c}^{A}(r) \geq 0$ holds, the retailer solves the following problem:

$$
\Pi_{r}^{T, A}=\max _{r \geq w_{r}}\left\{\Pi_{r}^{A}(r) \mid \Pi_{c}^{A}(r) \geq 0\right\}
$$

Because $\Pi_{c}^{A}(r)$ is increasing in the retailer's price $r$ as shown in $\S 5.1$, the constraint $\Pi_{c}^{A}(r) \geq 0$ holds if and only if the optimal $r$ that solves (37) satisfies $r \geq r^{D}$, where $r^{D}$ is characterized by (35). Observe from (36) that $\Pi_{r}^{A}(r)$ is a piecewise function in $r$, where the retailer can attract both high- and low-consumption consumers (when $w_{r} \leq r \leq \frac{v}{h}+w$ ), only low-consumption consumers (when $\frac{v}{h}+w \leq r \leq \frac{v}{l}+w$ ), or no consumers (when $r \geq \frac{v}{l}+w$ ). Let $r_{1}$ and $r_{2}$ denote the solution to the first-order conditions of $\Pi_{r}^{A}(r)$ in the cases where $r \in\left[w_{r}, \frac{v}{h}+w\right]$ and $r \in\left[\frac{v}{h}+w, \frac{v}{l}+w\right]$, respectively. Then it is easy to check from (36) that

$$
r_{1}=\frac{w+w_{r}}{2}+\frac{l(1-\alpha)+h \alpha}{2\left(l^{2}(1-\alpha)+h^{2} \alpha\right)} \cdot v \text { and } r_{2}=\frac{w+w_{r}}{2}+\frac{v}{2 l}
$$

Also, the corresponding $\Pi_{r}^{A}\left(r_{1}\right)=\frac{\left[\alpha h\left(v+h\left(w-w_{r}\right)\right)+(1-\alpha) l\left(v+l\left(w-w_{r}\right)\right)\right]^{2}}{8 v\left(l^{2}(1-\alpha)+h^{2} \alpha\right)}$, and $\Pi_{r}^{A}\left(r_{2}\right)=\frac{(1-\alpha)\left(v+l\left(w-w_{r}\right)\right)^{2}}{8 v}$. By comparing $\Pi_{r}^{A}\left(r_{1}\right)$ and $\Pi_{r}^{A}\left(r_{2}\right)$, we can further derive the optimal solution of the retailer's "tolerating problem" (37). As the structure of $\Pi_{r}^{A}(r)$ in (36) depends on parameters such as the coop's unit cost $w$, for ease of exposition, we shall focus on one of the cases in which $\Pi_{r}^{A}(r)$ takes the structure as illustrated in Figure $3{ }^{14}$ By solving (37), we show that in order to tolerate coop A's entry, the retailer should set the optimal price $r^{T, A}$ that satisfies the conditions in the following proposition. In preparation and for ease of exposition, we define $\kappa^{T} \equiv \Pi_{c}^{A}\left(r_{2}\right)+k$, which represents the coop's profit before the fixed annual operating cost $k$ is deducted, and note that $\kappa^{T}$ is independent of $k$.

Proposition 6. Suppose the retailer chooses to tolerate coop A's entry. Then, its optimal price $r^{T, A}$ is as follows:

${ }^{14}$ We focus on the case when the high-type consumer consumption quantity is large so that $h \geq \max \left\{3 l, 2 l \cdot \frac{1-\alpha}{1-2 \alpha}\right\}$ and the social-benefit level $v$ is high so that $\left(\sqrt{\frac{(h-l)^{2}(1-\alpha)}{h^{2} \alpha+l^{2}(1-\alpha)}}-1\right) \cdot v \geq h \cdot\left(w-w_{r}\right)$. And in this case, $r_{2} \in\left[\frac{v}{h}+w, \frac{v}{l}+w\right]$ and $\Pi_{r}^{A}\left(r_{2}\right) \geq \Pi_{r}^{A}\left(r_{1}\right)$. By using the same approach, we can derive the optimal solution to the retailer's problem for other cases as given in Online Appendix, and the deterrence strategy of coop A for other cases possesses the same structure as that in Proposition 7. 


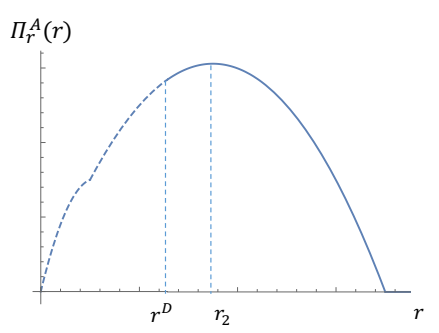

(a) $\boldsymbol{k} \leq \boldsymbol{\kappa}^{T}$

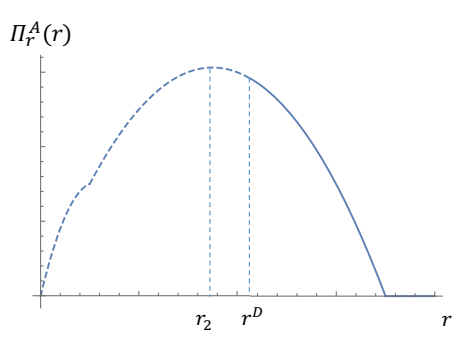

(b) $\kappa^{T} \in\left(\kappa^{T}, k_{2}\right]$

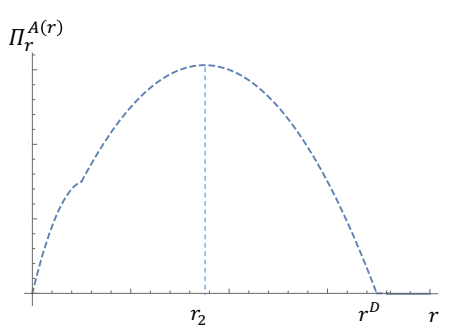

(c) $k>k_{2}$

Figure 3 The retailer profit under different ranges of the coop's fixed annual cost $k$. The solid curves represent the retailer profit when tolerating the coop. Setting: $\alpha=0.2, h=2, l=0.5, w=0.5, w_{r}=1, v=2$.

(a) If $k \leq \kappa^{T}$, then $r^{T, A}=r_{2} \equiv \frac{w+w_{r}}{2}+\frac{v}{2 l}$ so that the corresponding $\Pi_{r}^{T, A}=\Pi_{r}^{A}\left(r_{2}\right)=\frac{(1-\alpha)\left(v+l\left(w-w_{r}\right)\right)^{2}}{8 v}$. Also, $r^{T, A}$ and $\Pi_{r}^{T, A}$ are increasing in $\Delta w$.

(b) If $k \in\left(\kappa^{T}, k_{2}\right], r^{T, A}=r^{D}=w+\frac{2 \sqrt{k l^{2} v(1-\alpha)+h v^{2} \alpha(l(1-\alpha)+h \alpha)}-v(2 h \alpha+l(1-\alpha))}{l^{2}(1-\alpha)}$ so that the corresponding $\Pi_{r}^{T, A}=$ $\left(r^{D}-w_{r}\right) \cdot \frac{l(1-\alpha)}{2 v}\left(v-l\left(r^{D}-w\right)\right)$. Also, $r^{T, A}$ and $\Pi_{r}^{T, A}$ are increasing in $\Delta w$.

(c) If $k>k_{2}, r^{T, A}=r^{D}=w+\frac{k}{l(1-\alpha)+h \alpha}$ so that the corresponding $\Pi_{r}^{T, A}=0$. Also, $r^{T, A}$ is increasing in $\Delta w$.

Proposition 6 characterizes the optimal price $r^{T, A}$ that the retailer should charge when it chooses to tolerate the coop's entry, and implies that when $\Delta w$ is larger so that coop A's unit cost is higher, the retailer can afford to set a higher tolerating price $r^{T, A}$ so as to earn a higher profit $\Pi_{r}^{T, A}$. Proposition $6(\mathrm{a})$ shows that when coop A's annual operating cost $k$ is small, it is optimal for the retailer to set a certain tolerating price $r_{2}$ above the retailer's deterrence price $r^{D}$. Proposition $6(\mathrm{~b})-(\mathrm{c})$ shows that when $k$ is large, the retailer needs to set a high enough price $r^{T, A}=r^{D}\left(\geq r_{2}\right)$ to tolerate the coop's entry, and the tolerating price $r^{T, A}$ is increasing in $k$.

\subsection{Deter or Tolerate Coop A's Potential Entry}

We next determine whether the retailer should deter or tolerate coop A's entry by combining the results in Propositions 5 and 6. Recall from Proposition 5(a) that the retailer cannot deter the coop's entry when the coop's annual operating cost $k$ is very small (i.e., $k \leq k_{0}=\tau\left(w_{r}\right)$ ). On the other extreme, when $k$ is large (i.e., $\left.k>\kappa^{T}\right)$ so that $r^{T, A}=r^{D}$, Proposition 5(b) implies that the retailer can charge a slightly lower price than $r^{D}$ to deter coop A's entry, capture the entire market $(\alpha h+(1-\alpha) l)$, and increase its profit. Thus, when $k>\kappa^{T}$, the retailer's optimal strategy is to deter coop A's entry. In the following proposition, we shall focus on the more moderate values of $k$ (i.e., $k \in\left[k_{0}, \kappa^{T}\right]$ ), and obtain the retailer's optimal deterrence strategy by comparing its profit $\Pi_{r}^{D}$ when deterring the coop and its profit $\Pi_{r}^{T, A}$ when tolerating the coop.

Proposition 7. Suppose coop $A$ 's annual fixed cost $k>k_{0}$ so that the retailer is able to deter coop $A$ 's entry if so desired. Then there exists a threshold $\kappa_{A}<\kappa^{T}$ such that it is optimal for the retailer to:

1. Tolerate coop A's entry and set its selling price $r^{T, A}$ as described in Proposition 6 when $k \leq \kappa_{A}$;

2. Deter coop $A$ 's entry and set its selling price below $r^{D}$ as described in Proposition 5 when $k>\kappa_{A}$. Also, the threshold $\kappa_{A}$ is decreasing in $\Delta w$. 
Proposition 7 implies that the retailer should tolerate coop A's entry by setting its optimal selling price $r^{T, A}$ that satisfies $\Pi_{c}^{A}\left(r^{T, A}\right) \geq 0$ if and only if coop A's fixed annual operating cost $k$ is below a threshold $\kappa_{A}$. The intuition is as follows. Amid the threat of coop A's entry, the retailer faces a trade-off between the loss of profit due to charging a low deterrence price or the loss of profit due to losing some of its market share to the coop. When $k$ is low, coop A can afford to charge a low price and still obtain a positive profit. Thus, to deter the coop's entry, the retailer has to charge an even lower deterrence price $r^{D}$. Because the reduction in profit associated with the low deterrence price is much larger than the reduction in profit from losing some market share to the coop, the retailer is better off by tolerating coop A's entry. However, when $k$ is high, coop A can only afford to enter the market by setting a relatively high price. Hence, the retailer can deter coop A's entry by lowering its price slightly. In this case, the retailer's reduction in profit from losing some market share to coop A is larger than that from charging a deterrence price, so the retailer would prefer deterring coop A's entry. Furthermore, when coop A's unit cost is higher (i.e., $\Delta w$ is larger), it is more likely for the retailer to deter coop A's entry. This is because as the coop's unit cost becomes less competitive, the coop has to charge a higher price to stay profitable. Hence, the retailer can afford to set a higher deterrence price $r^{D}$ to deter coop A's entry without losing much profit.

\subsection{Deter or Tolerate Coop B's Potential Entry}

As discussed in $\S 5.1$, coop B cannot enter when the maximum profit that it can make, which equals coop A's maximum profit $\Pi_{c}^{A}(r)$, is below zero. Thus, Proposition 5 shows that when the coop's fixed annual operating cost $k>k_{0}$, the retailer can deter coop B's entry by setting a price slightly below $r^{D}$ and earn a profit slightly below $\Pi_{r}^{D}=\left(r^{D}-w_{r}\right) \cdot(\alpha h+(1-\alpha) l)$. We next examine whether the retailer should instead tolerate coop B's entry and set a higher price $r$ that satisfies $\Pi_{c}^{A}(r) \geq 0$. By anticipating coop B's entry and optimal pricing strategies, the retailer solves the following problem to find its optimal tolerating price:

$$
\Pi_{r}^{T, B}=\max _{r \geq w_{r}}\left\{\Pi_{r}^{B}(r) \mid \Pi_{c}^{A}(r) \geq 0\right\},
$$

where $\Pi_{r}^{B}(r)=\left(r-w_{r}\right) \cdot Q_{r}^{B}(r)$ is the retailer's profit when coop B adopts the optimal pricing strategy characterized in $\S 4.2$. Akin to coop A's problem given by (37), the retailer's tolerating price should satisfy $r \geq \max \left\{w_{r}, r^{D}\right\}$, where $r^{D}$ solves $\Pi_{c}^{A}\left(r^{D}\right)=0$ and is given by (35). Although it is analytically intractable to characterize the retailer's optimal tolerating price $r^{T, B}$, the following proposition characterizes the retailer's optimal deterrence strategy against coop B.

Proposition 8. Suppose coop B's fixed annual operating cost $k>k_{0}$ so that the retailer is able to deter or tolerate coop B's entry if so desired. Then there exist $\kappa_{B, 1}$ and $\kappa_{B, 2}$ such the retailer's optimal deterrence strategy against coop $B$ can be characterized as follows:

(a) When $k \leq \kappa_{B, 1}, \Pi_{r}^{T, B}>\Pi_{r}^{D}$, so it is optimal for the retailer to tolerate coop B's entry;

(b) When $k>\kappa_{B, 2}, \Pi_{r}^{T, B}<\Pi_{r}^{D}$, so it is optimal for the retailer to deter coop B's entry.

(c) The threshold $\kappa_{B, 2} \leq \kappa_{A}$, where $\kappa_{A}$ is as defined in Proposition \%. Hence, when it is optimal for the retailer to deter coop A, it is optimal for the retailer to deter coop $B$ as well. 
Proposition 8(a) shows that the retailer tolerates coop B when coop B's fixed annual operating cost $k$ is small (i.e., $\left.k \leq \kappa_{B, 1}\right)$ and Proposition $8(\mathrm{~b})$ shows that the retailer deters the coop when $k$ is large (i.e., $k>\kappa_{B, 2}$ ). Although Proposition 8 presents two thresholds, our numerical study shows and Figure 4 illustrates that these thresholds are actually equal, so there is a single threshold $\kappa_{B}=\kappa_{B, 1}=\kappa_{B, 2}$ such that it is optimal for the retailer to tolerate the coop if and only if $k \leq \kappa_{B} \cdot{ }^{15}$ This finding is in line with Proposition 7 , which shows that the retailer tolerates coop $\mathrm{A}$ if and only if $k \leq \kappa_{A}$ (we present a more general version of this proposition in Proposition EC.2 of Online Appendix), and these results have the same intuition. Proposition 8(c) implies that the threshold $\kappa_{B}$ is smaller than $\kappa_{A}$, so when $k \in\left(\kappa_{B}, \kappa_{A}\right)$, it is optimal for the retailer to tolerate coop A's entry but deter coop B's entry; see Figure 4. This result can be explained by using Corollary 2, which reveals that coop B's entry is more detrimental to the retailer than coop A's entry. Accordingly, the retailer is more aggressive against coop B's entry. This result shows that a retailer and a coop with social mission (A) are more likely to co-exist than a retailer and a coop with social mission (B).

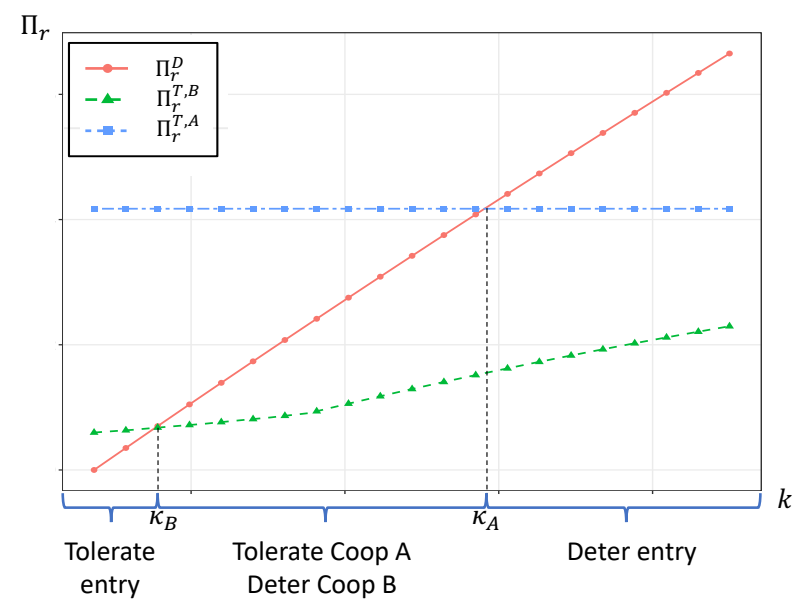

Figure 4 The retailer's profit $\Pi_{r}^{D}$ when deterring the coop (A or B), $\Pi_{r}^{T, A}$ when tolerating coop A, and $\Pi_{r}^{T, B}$ when tolerating coop B as a function of the coop's annual operating cost $k$. Setting: $\alpha=0.8, l=0.5$, $h=2, w=0.5, w_{r}=1, v=5.5$.

Analogous to retailer's deterrence strategy against coop A as given by Proposition 7, Figure 5 shows that the threshold $\kappa_{B}$ for coop B is also decreasing in $\Delta w$, which reveals that the retailer will be more aggressive against the coop with higher unit cost regardless of its social mission. This result implies that if the coop can further decrease its unit cost, then it will be more likely to enter the market and coexist with the retailer.

\footnotetext{
${ }^{15}$ In our extensive numerical study, we have randomly generated 10,000 instances, and at each instance, we have randomly selected the proportion of high-consumption consumers $\alpha$ from Uniform[0,1], the high-consumption amount $h$ from Uniform $[2,10]$, the low-consumption amount $l$ from Uniform[1,h], the social-benefit level as $v$ from Uniform[2,10], the coop's unit cost $w$ from Uniform $[0,2]$, and the retailer's unit cost $w_{r}$ from Uniform $\left[\max \left\{0, w-\frac{v}{h}\right\}, w+\frac{v}{h}\right]$. We have increased the coop's fixed annual operating cost $k$, and tested whether the retailer's profit $\Pi_{r}^{D}$ when deterring the coop increases at a faster rate than its profit $\Pi_{r}^{T, B}$ when tolerating coop B as depicted in Figure 4 . At all instances, $\Pi_{r}^{D}$ increased faster with $k$ than $\Pi_{r}^{T, B}$. This result combined with Proposition 8 indicates that there should exist a unique threshold $\kappa_{B}$ such that it is optimal for the retailer to tolerate the coop if and only if $k \leq \kappa_{B}$.
} 


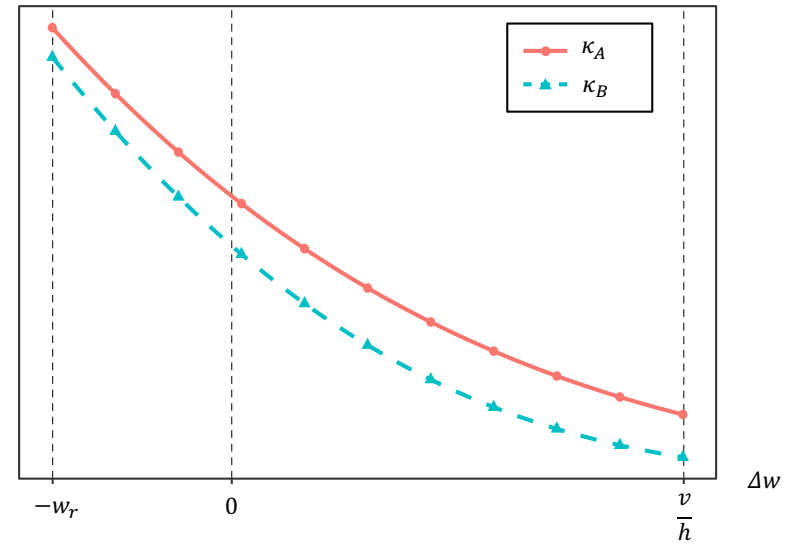

Figure $5 \quad \kappa_{A}$ and $\kappa_{B}$ when $\Delta w \in\left[-w_{r}, \frac{v}{h}\right]$ (i.e., $\left.w \in\left[0, w_{r}+\frac{v}{h}\right]\right)$. Setting: $\alpha=0.8, l=0.5, h=2, w_{r}=1, v=5.5$.

\section{Conclusion}

As a response to a rallying cry for the support of local communities and local economies, an increasing number of not-for-profit food coops establish their presence in less populated areas or poor communities in recent years. This trend has motivated us to examine the entry conditions for food coops with different social missions: (A) Maximize profit to support local community needs; and (B) Maximize sales to support local economy. To this end, we have analyzed a 2-stage Stackelberg competition between an incumbent forprofit retailer and an entrant coop in a market comprised of heterogeneous consumers with different annual consumption rates and social benefit levels. We have examined the coop's entry conditions, the impact of the coop's entry on the retailer's sales and profit, and the conditions under which the retailer should deter the coop's entry.

We have first analyzed the coop's entry conditions and its competition with the retailer and found that a coop with mission (A) or (B) can only afford to enter the market when the social-benefit level is sufficiently high. Interestingly, we found that a high social-benefit level also helps the retailer to survive against coop with social mission (A), but it weakens the retailer's survival against the coop with social mission (B). In the latter case, the retailer and the coop can co-exist only when the coop's annual operating cost takes moderate values. When the coop can enter the market, we have shown that the coop (regardless of its social mission) sets its membership fee and member-only discount to induce at least the high-consumption consumers to become members.

We have also examined the impact of a coop's entry on the retailer, and whether the retailer should deter or tolerate the coop's entry. Interestingly, we have found that the retailer should tolerate the coop's entry when the coop's cost of entry is below a certain threshold. We further show that this threshold is smaller for a coop with social mission (B) than it is for a coop with social mission (A), so the retailer is less tolerant against the coop with social mission (B). This interesting result is driven by the fact that the entry of a coop with social mission (B) is more detrimental to the retailer's profit than the entry of a coop with social mission (A).

As an initial attempt to examine the entry of a not-for-profit coop with a social mission and the impact of its entry on the incumbent for-profit retailer, we hope our paper can serve as catalyst for future research. 
First, for tractability, we have assumed that the consumption rate of each consumer is exogenously given and independent of price. This is reasonable for a product that is not very price sensitive or a product with stable consumption rate. Examples include tooth paste, toilet paper, milk, bread, etc. However, for products that are highly price sensitive, it is of interest to examine the case when the consumption rate is endogenously dependent on the selling price. Second, we treat missions (A) and (B) separately to isolate their effects and to ensure tractability, yet some coops may consider both missions simultaneously. Furthermore, some coops may possess other social missions such as focusing on organic food products or supporting a particular affinity group (e.g., women and minority). Third, for tractability, we invoke the notion of irreversibility as a committed threat to deter entry, so the retailer does not change its price after the entry of a coop. Although this notion is standard in the market-entry framework, it may be of some interest to extend our analysis to a case where the retailer and the coop set their selling price simultaneously.

\section{References}

Adams, D. 2020. Food co-ops, the future is now. Nonprofit Quarterly https://nonprofitquarterly.org/ for-food-co-ops-the-future-is-now/. Accessed on November 12, 2020.

Aghion, P., P. Bolton. 1987. Contracts as a barrier to entry. The American Economic Review 77(3) 388-401.

An, J., S. Cho, C. S. Tang. 2015. Aggregating smallholder farmers in emerging economies. Production and Operations Management 24(9) 1414-1429.

Andreoni, J. 1990. Impure altruism and donations to public goods: A theory of warm-glow giving. The economic journal 100(401) 464-477.

Ayvaz-Cavdaroğlu, N., B. Kazaz, S. Webster. 2020. Incentivizing farmers to invest in quality through qualitybased payment. Working paper, Syracuse University, New York.

Bain, J. S. 1949. A note on pricing in monopoly and oligopoly. The American Economic Review 39(2) $448-464$.

Baron, D. P. 1973. Limit pricing, potential entry, and barriers to entry. The American Economic Review 63(4) 666-674.

Berg, J. 2011. Turning food deserts into jobs oases. Huffington Post, https://www.huffpost.com/entry/ turning-food-deserts-into_b_384321, Accessed on November 11, 2020.

Brinkley, C. 2019. Why community-owned grocery stores like co-ops are the best recipe for revitalizing food deserts https://theconversation.com/ why-community-owned-grocery-stores-like-co-ops-are-the-best-recipe-for-revitalizing-food-deserts- 122997. Accessed on November 12, 2020.

Brooks, K. 2014. Research shows food desserts more abundant in minority neighborhoods. Johns Hopkins Magazine, https://hub.jhu.edu/magazine/2014/spring/racial-food-deserts. Accessed on November 11, 2020.

Brzyski, L. 2019. Food co-ops are on the rise in Philly https://www.phillymag.com/be-well-philly/2019/10/ 23/philly-food-co-ops/. Accessed on November 12, 2020. 
Catlette, M. 2012. Food co-ops' community impact. https://www.grocer.coop/articles/numbers. Accessed on August 24, 2020.

Chen, G., C. G. Korpeoglu, S. E. Spear. 2017. Price stickiness and markup variations in market games. Journal of Mathematical Economics 72 95-103.

Corbett, C. J., U. S. Karmarkar. 2001. Competition and structure in serial supply chains with deterministic demand. Management Science 47(7) 966-978.

Deller, S., A. Hoyt, B. Hueth, R. Sundaram-Stukel. 2009. Research on the economic impact of cooperatives. University of Wisconsin Center for Cooperatives 231 232-233.

Elliott, M. 2020. African heritage food co-op continues to grow produce and food model https://spectrumlocalnews.com/nys/buffalo/news/2020/06/11/ african-heritage-food-co-op-continues-to-grow-produce-and-their-food-model-. Accessed on November 11, 2020.

Fudenberg, D., J. Tirole. 1991. Game theory. MIT press.

Gao, S. Y., W. S. Lim, C. S. Tang. 2017. Entry of copycats of luxury brands. Marketing Science 36(2) $272-289$.

Gulker, M. 2018. Food coops and capitalism. https://www.aier.org/article/food-co-ops-and-capitalism. Accessed on August 24, 2020.

Hall, R. E. 2008. Potential competition, limit pricing, and price elevation from exclusionary conduct. Issue in Competition Law and Policy 433 433-448.

Halliday, L., M. Foster. 2020. A tale of two co-ops in two cities. Journal of Agriculture, Food Systems, and Community Development 9(2) 239-254.

Harbaugh, W. T. 1998. What do donations buy?: A model of philanthropy based on prestige and warm glow. Journal of Public Economics 67(2) 269-284.

He, Q.-C., Y-J. Chen, Z.-J. Shen. 2018. On the formation of producers' information-sharing coalitions. Production and Operations Management 27(5) 917-927.

Ide, E., J-P Montero, N. Figueroa. 2016. Contracts as a barrier to entry. The American Economic Review 106(7) 1849-1877.

Jackson, J. 2019. Price comparison: Your co-op vs. retail food giants. https://communityfood.coop/ price-comparison-your-co-op-vs-retail-food-giants. Accessed on March 28, 2020.

Karpyn, A. E., D. Riser, T. Tracy, R. Wang, Y. E. Shen. 2019. The changing landscape of food deserts. United Nations System Standing Committee on Nutrition 44 46-53.

Kauffman, J. 2017. The rise of the modern food cooperative. https://www.sfgate.com/news/article/ The-rise-of-the-modern-food-cooperative-11006896.php. Accessed on March 29, 2020.

Knupfer, A. M. 2013. Food co-ops in America: communities, consumption, and economic democracy. Cornell University Press.

Korpeoglu, C G., E. Körpeoğlu, S.-H. Cho. 2020. Supply chain competition: A market game approach. Management Science 66(12) 5648-5664. 
McCardle, K. F., K. Rajaram, C. S. Tang. 2009. A decision analysis tool for evaluating fundraising tiers. Decision Analysis 6(1) 4-13.

Milgrom, P., J. Roberts. 1982. Limit pricing and entry under incomplete information: An equilibrium analysis. Econometrica 50(2) 443-459.

Mu, L., M. Dawande, V. Mookerjee. 2019. Shaping the values of a milk cooperative: Theoretical and practical considerations. Production and Operations Management 28(9) 2259-2278.

Nalebuff, B. 2004. Bundling as an entry barrier. Quarterly Journal of Economics 119(1) 159-187.

Nargi, L. 2020. Community food coops are thriving during the pandemic https://civileats.com/2020/05/15/ community-food-co-ops-are-thriving-during-the-pandemic/. Accessed on November 11, 2020.

Nelson, P. 2020. Schenectady plan for community-owned grocery moving forward. https://www.timesunion. com/news/article/Schenectady-coop-eyeing-two-possible-sites-for-15099548.php. Accessed on August $24,2020$.

Nembhard, J. G. 2014a. The benefits and impacts of cooperatives. https://geo.coop/story/ benefits-and-impacts-cooperatives. Accessed on August 24, 2020.

Nembhard, J. G. 2014b. Collective courage: A history of African American cooperative economic thought and practice. Penn State University Press, Unversity Park, PA .

Peters, A. 2018. To end a food desert, these community members opened their own grocery. Fast Company https://www.fastcompany.com/40514428/ to-end-a-food-desert-these-community-members-opened-their-own-grocery. Accessed on November $11,2020$.

Picchi, A. 2015. Walmart's china syndrome: The loss of us jobs. CBS News https://www.cbsnews.com/ news/walmarts-china-syndrome-the-loss-of-us-jobs. Accessed on March 27, 2020.

Qian, X., T. L. Olsen. 2020. Operational and financial decisions within proportional investment cooperatives. Manufacturing \& Service Operations Management 22(3) 545-561.

Roberts, N. 2019. New generation of black-led co-ops want to end food insecurity. The Root https:// www.theroot.com/new-generation-of-black-led-co-ops-want-to-end-food-ins-1840411111., Accessed on November 11, 2020.

Seattle, J. 2020. With vow to prevent retail racism, pcc opens in the central district https://www. capitolhillseattle.com/2020/06/with-vow-to-prevent-retail-racism-pcc-opens-in-the-central-district/. Accessed on November 11, 2020.

Shi, J., Y. Zhao, R. B. K. Kiwanuka, J. Chang. 2019. Optimal selling policies for farmer cooperatives. Production and Operations Management 28(12) 3060-3080.

Spence, A. M. 1977. Entry, capacity, investment and oligopolistic pricing. The Bell Journal of Economics $534-544$.

Spence, A. M. 1979. Investment strategy and growth in a new market. J. Reprints Antitrust L. E Econ. 10 345. 
Srinivasan, K. 1991. Multiple market entry, cost signalling and entry deterrence. Management Science 37(12) $1539-1555$.

Wilbourn, M. S. 2015. Bringing grocery stores to low-income urban food deserts. https://resources.uwcc. wisc.edu/Grocery/grocery_low-income_urban_food_deserts.pdf.

\section{Appendix: Proofs}

Proof of Proposition 1. The proof proceeds as follows. We first determine the optimal pricing decisions $\left(F_{i}^{A}, p_{i}^{A}, \delta_{i}^{A}\right)$ of coop A under pricing strategies 1 and 2, and show that each statement of the proposition holds under each of these strategies. Then we show that strategy 3 is dominated by strategies 1 and 2 , so we can eliminate strategy 3 . Thus, it will follow that the statements of the proposition hold regardless of whether coop A chooses strategy 1 or 2 .

Pricing strategy 1: From (15), we obtain the Hessian matrix of the objective function as:

$$
\mathbf{H}_{\mathbf{1}}=\left[\begin{array}{cc}
-\frac{2}{v} & -\frac{2(h \alpha+l(1-\alpha))}{v} \\
-\frac{2(h \alpha+l(1-\alpha))}{v} & -\frac{2\left(h^{2} \alpha+l^{2}(1-\alpha)\right)}{v}
\end{array}\right] .
$$

As such, for any given vector $\mathbf{x}=\left[x_{1}, x_{2}\right]^{T} \in \mathbb{R}_{++}^{2}$, we can check that $\mathbf{x}^{T} \mathbf{H}_{1} \mathbf{x}=-\frac{2}{v}\left[\left(x_{1}+(l(1-\alpha)+\right.\right.$ $\left.\left.h \alpha) x_{2}\right)^{2}+\alpha(1-\alpha)(h-l)^{2} x_{2}^{2}\right]<0$. Thus, the Hessian matrix is negative definite. By solving $\frac{\partial \Pi_{c 1}}{\partial F}=0$ and $\frac{\partial \Pi_{c 1}}{\partial \tilde{p}}=0$ simultaneously, we obtain $F^{*}=\frac{v}{2}$ and $\tilde{p}^{*}=\frac{r+w}{2}$, which are optimal if feasible. We next check the feasibility of $\left(F^{*}, \tilde{p}^{*}\right)=\left(\frac{v}{2}, \frac{r+w}{2}\right)$. Note that $\left(F^{*}, \tilde{p}^{*}\right)$ satisfies $F^{*} \geq 0$ and $\tilde{p}^{*} \geq 0$.

1. If $r \geq w$, then $\frac{v}{2}+\frac{r-w}{2} \cdot l \geq 0$ and $\frac{v}{2}+\frac{r-w}{2} \cdot h \geq 0$ so $\left(F^{*}, \tilde{p}^{*}\right)$ satisfies constraints (18) and (19).

(a) If $v>(r-w) h$, we can check that $F^{*}+\left(\tilde{p}^{*}-r\right) \cdot l=\frac{v}{2}-\frac{r-w}{2} \cdot l \geq 0$ and $F^{*}+\left(\tilde{p}^{*}-r\right) \cdot h=$ $\frac{v}{2}-\frac{r-w}{2} \cdot h \geq 0$. Therefore, $\left(F_{1}^{A}, \tilde{p}_{1}^{A}\right)=\left(\frac{v}{2}, \frac{r+w}{2}\right)$ is optimal and the corresponding profit $\Pi_{c 1}^{A}=$ $\frac{1}{4 v}\left[(1-\alpha)(v+l(r-w))^{2}+\alpha(v+h(r-w))^{2}\right] \equiv \tau-k$, where $\tau$ is defined in (21).

(b) If $v \in((r-w) l,(r-w) h],\left(F^{*}, \tilde{p}^{*}\right)$ violates $(17)$. By setting $F+(\tilde{p}-r) h=0$, we obtain the corner solution $\left(F_{1}^{A}, \tilde{p}_{1}^{A}\right)=\left(\frac{h(v-l(r-w))}{2(h-l)}, \frac{2 h r-l r-v-l w}{2(h-l)}\right)$, and the corresponding profit $\Pi_{c 1}^{A}=$ $\tau-\frac{\alpha(v-h(r-w))^{2}}{4 v}-k$, so coop A captures the whole high-consumption consumer market.

(c) if $v \in[0,(r-w) l],\left(F^{*}, \tilde{p}^{*}\right)$ violates (16) and (17). Thus, the corner solution $F_{1}^{A}=0$ and $\tilde{p}_{1}^{A}=r$ is optimal, and the corresponding profit $\Pi_{c 1}^{A}=\tau-\frac{\alpha(v-h(r-w))^{2}}{4 v}-\frac{(1-\alpha)(v-l(r-w))^{2}}{4 v}-k$, so coop A captures the whole market.

2. If $r<w$, then $\frac{v}{2}-\frac{r-w}{2} \cdot l \geq 0$ and $\frac{v}{2}-\frac{r-w}{2} \cdot h \geq 0$ so that $\left(F^{*}, \tilde{p}^{*}\right)$ satisfies (16) and (17).

(a) If $v>(w-r) \cdot h,\left(F^{*}, \tilde{p}^{*}\right)$ also satisfies (18) and (19) so that the corresponding $\Pi_{c 1}^{A}=\tau-k$.

(b) If $v \in((w-r) \cdot l,(w-r) \cdot h],\left(F^{*}, \tilde{p}^{*}\right)$ violates $(18)$. By setting $v-(F+(\tilde{p}-r) \cdot h)=0$, we obtain the corner solution $\left(F_{1}^{A}, \tilde{p}_{1}^{A}\right)=\left(\frac{-2 l v+h(v+l(w-r))}{2(h-l)}, \frac{2 h r-l r+v-l w}{2(h-l)}\right)$ and the profit $\Pi_{c 1}^{A}=$ $\frac{(1-\alpha)(v-l(w-r))^{2}}{4 v}-k$, so the retailer captures the whole high-consumption consumer market. 
(c) If $v \in[0,(w-r) \cdot l],\left(F^{*}, \tilde{p}^{*}\right)$ violates (18) and (19). By setting $v-(F+(\tilde{p}-r) \cdot h)=0$ and $v-(F+(\tilde{p}-r) \cdot l)=0$, we obtain the corner solution $\left(F_{1}^{A}, \tilde{p}_{1}^{A}\right)=(v, r)$ so that $\Pi_{c 1}^{A}=-k$ and the retailer captures the whole market.

Thus, we can obtain that when coop A adopts Strategy 1, the corresponding market shares of coop A and the retailer under different $v$ are as depicted in Figure 2. Based on the optimal $\left(F_{1}^{A}, p_{1}^{A}\right)$ under the above cases, we can obtain the corresponding $\delta_{1}^{A}$ that satisfies $\delta_{1}^{A} \geq \frac{F_{1}^{A}}{l}$ and $p_{1}^{A}=\tilde{p}_{1}^{A}+\delta_{1}^{A}$.

Pricing strategy 2: From (22), we obtain the Hessian matrix of the objective function as:

$$
\mathbf{H}_{2}=\left[\begin{array}{cc}
-\frac{2 \alpha}{v} & -\frac{2 h \alpha}{v} \\
-\frac{2 h \alpha}{v} & -\frac{2\left(l^{2}(1-\alpha)+h^{2} \alpha\right)}{v}
\end{array}\right]
$$

As such, for any given $\mathbf{x}=\left[x_{1}, x_{2}\right]^{T} \in \mathbb{R}_{++}^{2}$, we can check that $\mathbf{x}^{T} \mathbf{H}_{2} \mathbf{x}=-\frac{2 \alpha}{v}\left(x_{1}+h x_{2}\right)^{2}-$ $\frac{2 l^{2}(1-\alpha)}{v} x_{2}^{2}<0$. The Hessian matrix is negative definite. By solving $\frac{\partial \Pi_{c 2}}{\partial \tilde{F}}=0$ and $\frac{\partial \Pi_{c 2}}{\partial p}=0$ simultaneously, we obtain $\tilde{F}^{*}=-\frac{(h-l) v}{2 l}$ and $p^{*}=\frac{r+w}{2}+\frac{v}{2 l}$, which are optimal if feasible. We shall check the feasibility of $\left(\tilde{F}^{*}, p^{*}\right)=\left(-\frac{(h-l) v}{2 l}, \frac{r+w}{2}+\frac{v}{2 l}\right)$. First, we can check that $\left(\tilde{F}^{*}, p^{*}\right)$ satisfies $(27)$.

1. If $r \geq w$, then we can obtain that $\left(\tilde{F}^{*}, p^{*}\right)$ satisfies (25) and (26).

(a) If $v>(r-w) \cdot h,\left(\tilde{F}^{*}, p^{*}\right)=\left(-\frac{(h-l) v}{2 l}, \frac{r+w}{2}+\frac{v}{2 l}\right)$ also satisfies (23) and (24). Therefore, $\left(\tilde{F}_{2}^{A}, p_{2}^{A}\right)=\left(\tilde{F}^{*}, p^{*}\right)=\left(-\frac{(h-l) v}{2 l}, \frac{r+w}{2}+\frac{v}{2 l}\right)$ is optimal and the corresponding profit $\Pi_{c 2}^{A}=\tau-k$.

(b) If $v \in((r-w) l,(r-w) h],\left(\tilde{F}^{*}, p^{*}\right)$ violates $(23)$. By setting $\tilde{F}+(p-r) \cdot h=0$, we obtain the corner solution $\left(\tilde{F}_{2}^{A}, p_{2}^{A}\right)=\left(h\left(r-\frac{v+l(r+w)}{2 l}\right), \frac{r+w}{2}+\frac{v}{2 l}\right)$ so that the corresponding $\Pi_{c 2}^{A}=$ $\tau-\frac{\alpha(v-(r-w) h)^{2}}{4 v}-k$ and coop A captures the whole high-consumption consumer market.

(c) If $v \in[0,(r-w) l]$, we obtain the corner solution $\left(\tilde{F}_{2}^{A}, p_{2}^{A}\right)=(0, r)$ so that the corresponding $\Pi_{c 2}^{A}=\tau-\frac{\alpha(v-(r-w) h)^{2}}{4 v}-\frac{(1-\alpha)(v-(r-w) l)^{2}}{4 v}-k$. In this case, coop A captures the whole market. 2. If $r<w$, then we can check that $\left(\tilde{F}^{*}, p^{*}\right)=\left(-\frac{(h-l) v}{2 l}, \frac{r+w}{2}+\frac{v}{2 l}\right)$ always satisfies (23) and (24).

(a) If $v>(w-r) \cdot h,\left(\tilde{F}^{*}, p^{*}\right)$ satisfies (25) and (26) so that the corresponding $\Pi_{c 2}^{A}=\tau-k$.

(b) If $v \in((w-r) \cdot l,(w-r) \cdot h],\left(\tilde{F}^{*}, p^{*}\right)$ violates $(18)$. By setting $v-(\tilde{F}+(p-r) \cdot h)=0$, we obtain the corner solution $\left(\tilde{F}_{2}^{A}, p_{2}^{A}\right)=\left(v+\frac{h}{2}\left(r-w+\frac{v}{l}\right), \frac{r+w}{2}+\frac{v}{2 l}\right)$ so that $\Pi_{c 2}^{A}=\frac{(1-\alpha)(v+l(r-w))^{2}}{4 v}-k$ and the retailer captures the whole high-consumption consumer market.

(c) $v \in[0,(w-r) \cdot l],\left(\tilde{F}^{*}, p^{*}\right)$ violates $(25)$ and $(26)$. By setting $v-(\tilde{F}+(p-r) \cdot h)=0$ and $v-(p-r) \cdot l=0$, we obtain $\left(\tilde{F}_{2}^{A}, p_{2}^{A}\right)=\left(-(h-l) \cdot \frac{v}{l}, \frac{v}{l}+r\right)$ so that $\Pi_{c 2}^{A}=-k$. In this case, the retailer captures the whole market.

Therefore, when coop A adopts strategy 2, the corresponding market shares of coop A and the retailer under different $v$ are the same as those under strategy 1, and as depicted in Figure 2. Based on the optimal $\left(F_{1}^{A}, p_{1}^{A}\right)$ under the above cases, we can further obtain the corresponding $\delta_{2}^{A}$ that satisfies $\delta_{2}^{A} \in\left[-\frac{\tilde{F}_{2}^{A}}{h-l}, p_{2}^{A}\right]$ so that the corresponding $F_{2}^{A}=\tilde{F}_{2}^{A}+\delta_{2}^{A}$.

Pricing strategy 3: From (12), we know that when coop A adopt strategy 3, it solves:

$$
\Pi_{c 3}^{A}=\max _{p \geq w}\left(\frac{\alpha}{v} \cdot[v-(p-r) \cdot h] \cdot(p-w) h+\frac{1-\alpha}{v} \cdot[v-(p-r) \cdot l] \cdot(p-w) l\right)-k
$$




$$
\begin{aligned}
& \text { s.t. (24), (26), } \\
& v-(p-r) \cdot h \geq 0 .
\end{aligned}
$$

By checking the first- and second-order conditions, we obtain that $\frac{\partial \Pi_{c 3}}{\partial p}=\frac{\alpha}{v} \cdot h(v-h(2 p-r-$ $w))+\frac{(1-\alpha)}{v} \cdot l(v-l(2 p-r-w))$ and $\frac{\partial^{2} \Pi_{c 3}}{\partial p^{2}}=-\frac{2}{v}\left(\alpha h^{2}+(1-\alpha) l^{2}\right)<0$. Therefore, by setting $\frac{\partial \Pi_{c 3}}{\partial p}=0$, we obtain that the optimal $p_{c 3}^{A}=p^{*}=\frac{\alpha h[v+h(w+r)]+(1-\alpha) l[v+l(w+r)]}{2\left(\alpha \cdot h^{2}+(1-\alpha) \cdot l^{2}\right)}$ when $p^{*}$ is feasible. By substituting the $p^{*}$ into (10) and (12), we can further obtain the corresponding $\Pi^{*}=\tau^{\prime}-k \equiv$ $\frac{[l(v+l(r-w))(1-\alpha)+h(v+h(r-w)) \alpha]^{2}}{4 v\left[(1-\alpha) l^{2}+\alpha h^{2}\right]}-k \leq \tau-k$. Also, in pricing strategy 3, as $(F, \delta)$ are no longer the relevant decisions, by setting $(F, \delta, p)=\left(0,0, p_{3}^{B}\right)$, we can check that it is also a feasible solution of pricing strategy 1 and 2. Therefore, pricing strategy 3 is dominated by strategy 1 and 2. Q.E.D.

Proof of Lemma 1. From the analysis of coop A's pricing strategy 1 in the proof of Proposition 1, we know that when $v \geq|r-w| \cdot h$, the optimal $\left(F_{1}^{A}, \tilde{p}_{1}^{A}\right)=\left(\frac{v}{2}, \frac{r+w}{2}\right)$ so that the corresponding $\left(F_{1}^{A}, p_{1}^{A}, \delta_{1}^{A}\right)$ are as given by Lemma 1 . By substituting $\left(F_{1}^{A}, p_{1}^{A}, \delta_{1}^{A}\right)$ into $Q_{c 1}$ as given by (2) and $\Pi_{c 1}$ as given by (4), we can obtain the corresponding $Q_{c 1}^{A}$ and $\Pi_{c 1}^{A}$ as given by Lemma 1. Q.E.D. Proof of Corollary 1. Given the retailer's unit cost as $w_{r}$ and coop A's unit cost $w=w_{r}+\Delta w$, it is possible that $\Delta w \leq 0$ (Scenario (a)) and $\Delta w>0$ (Scenario (b)). Hence, we can use the following figure $6(\mathrm{a})$ and $6(\mathrm{~b})$ to illustrate different cases when the retailer charges $r \geq w_{r}$ (shaded area). Using Proposition 1 and by observing Figure 6, we can easily obtain that given the retailer's price

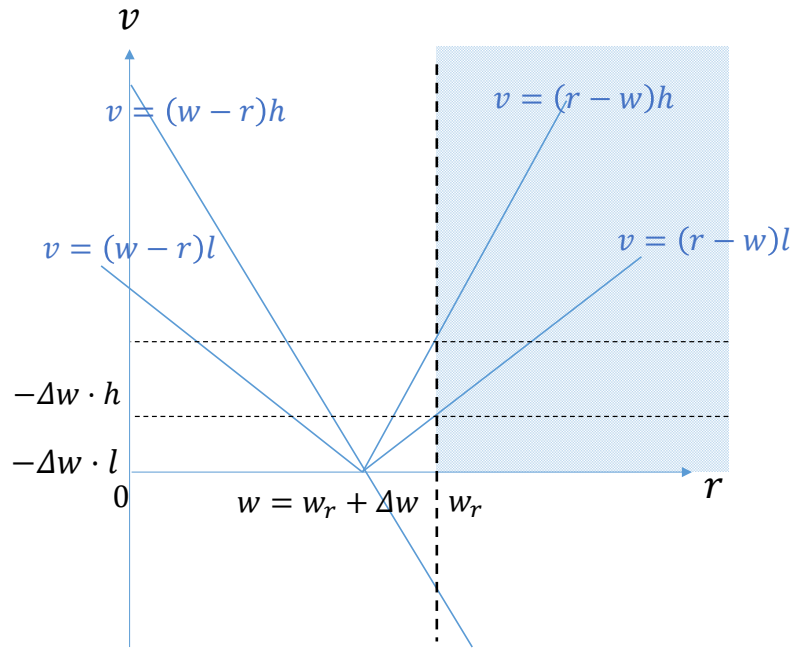

(a): $\Delta w \leq 0\left(\right.$ i.e.,$\left.w \leq w_{r}\right)$

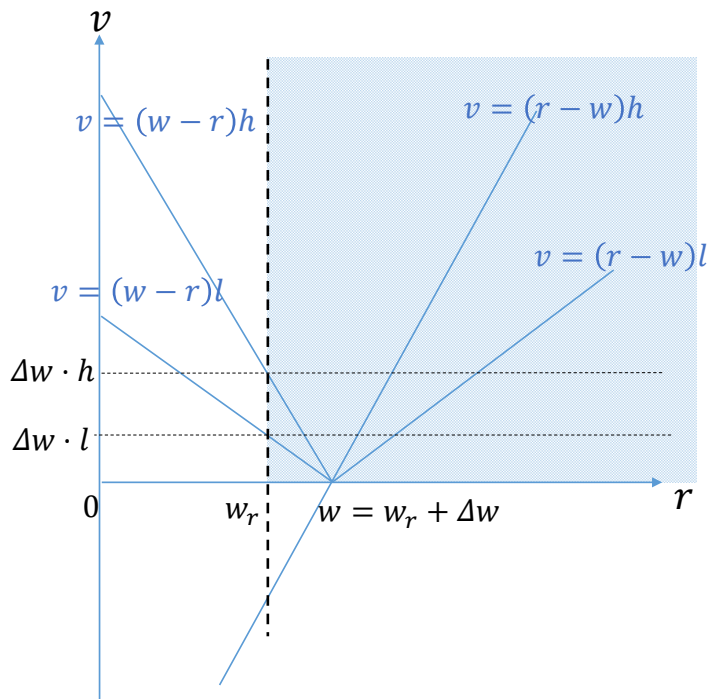

(b): $\Delta w>0\left(\right.$ i.e., $\left.w>w_{r}\right)$

Figure 6 Scenario (a): when $\Delta w \leq 0$ and Scenario (b): when $\Delta w>0$. 
$r \geq w_{r}$ and coop A's unit cost $w=w_{r}+\Delta w$, the market shares of the retailer and the coop satisfy the following properties:

(a). Suppose $\Delta w \leq 0$.

- if $v>-\Delta w \cdot h$, then it is possible that coop A may (1) capture the whole market, (2) capture the whole high-consumption consumer market, or (3) share both high- and low-consumption consumer markets with the retailer;

- if $v \in(-\Delta w \cdot l,-\Delta w \cdot h]$, then it is possible that coop A may (1) capture the whole market or (2) capture the whole high-consumption consumer market;

- if $v \leq-\Delta w \cdot l$, then coop A can always capture the whole market.

(b). Suppose $\Delta w>0$. and

- if $v>\Delta w \cdot h$, then it is possible that coop A may (1) capture the whole market, (2) capture the whole high-consumption consumer market, or (3) share both high- and low-consumption consumer markets with the retailer;

- if $v \in(\Delta w \cdot l, \Delta w \cdot h]$, besides scenarios (1), (2), (3), it is also possible that coop A may (4) get part of the market share only from the low-consumption consumer market and scenario (4) occurs when $r \leq w_{r}+\Delta w-\frac{v}{h}$;

- if $v \leq \Delta w \cdot l$, besides scenarios (1), (2), (3), (4), it is also possible that (5) coop A cannot get any market share from any market and scenario (5) occurs when $r \leq w_{r}+\Delta w-\frac{v}{l}$.

Proof of Lemma 2. From the analysis of coop A's pricing strategy 2 in the proof of Proposition 1 , we know that when $v \geq|r-w| \cdot h$, the optimal $\left(\tilde{F}_{2}^{A}, p_{2}^{A}\right)=\left(-\frac{(h-l) v}{2 l}, \frac{r+w}{2}+\frac{v}{2 l}\right)$ so the corresponding $\left(F_{2}^{A}, p_{2}^{A}, \delta_{2}^{A}\right)$ are as given by Lemma 2 . By substituting $\left(F_{2}^{A}, p_{2}^{A}, \delta_{2}^{A}\right)$ into $Q_{c 2}$ as given by (6) and $\Pi_{c 2}$ as given by (8), we can obtain the corresponding $Q_{c 2}^{A}$ and $\Pi_{c 2}^{A}$ as given by Lemma 2. Q.E.D.

Proof of Lemma 3. See the analysis of pricing strategy 3 in the proof of Proposition 1. Q.E.D.

Proof of Proposition 2. From the proof of Proposition 1, we know that it is optimal for coop A to adopt strategy 1 or 2 , and the maximum profit is $\tau-k$ when $v \geq|r-w| \cdot h$. Therefore, coop A can afford to enter the market only when $k \leq \tau$, where $\tau$ is as given by (21). Q.E.D.

Proof of Proposition 3. First, from Proposition 2, we know that when $v \geq|r-w| \cdot h$, the maximum profit that the coop can make is $\Pi_{c}^{A}=\tau-k$. Thus, to ensure that coop B can enter the market, we should have the condition $k \leq \tau$. Given $k \leq \tau$, the rest of the proof proceeds as follows. We first determine the optimal pricing decision $\left(F_{i}^{B}, p_{i}^{B}, \delta_{i}^{B}\right)$ of coop B under pricing strategies 1 and 2 , and show that each statement of the proposition holds under each strategy. Then we show that strategy 3 is dominated by strategies 1 and 2 , hence can be eliminated. Thus, it will follow that the statements of the proposition hold regardless of whether coop A chooses strategy 1 or 2 . 
Pricing strategy 1: As maximizing $Q_{c 1}$ is equivalent to minimizing $-Q_{c 1}$, we can write the Lagrangian Relaxation of the coop B's problem under strategy 1 as:

$$
\begin{gathered}
L(\lambda)=\min _{F, \tilde{p}}-Q_{c 1}(F, \tilde{p})+\lambda \cdot\left(0-\Pi_{c 1}(F, \tilde{p})\right) \\
\text { s.t. }(16),(17),(18),(19),(20) .
\end{gathered}
$$

The Hessian matrix of the objective function is $\mathbf{H}_{\mathbf{1}}=\left[\begin{array}{cc}\frac{2 \lambda}{v} & \frac{2 \lambda(h \alpha+l(1-\alpha))}{v} \\ \frac{2 \lambda(h \alpha+l(1-\alpha))}{v} & \frac{2 \lambda\left(h^{2} \alpha+l^{2}(1-\alpha)\right)}{v}\end{array}\right]$. For any given vector $\mathbf{x}=\left[x_{1}, x_{2}\right]^{T} \in \mathbb{R}_{++}^{2}$, we can check that $\mathbf{x}^{T} \mathbf{H}_{1} \mathbf{x}=\frac{2 \lambda}{v}\left[\left(x_{1}+(l(1-\alpha)+h \alpha) x_{2}\right)^{2}+\alpha(1-\alpha)(h-\right.$ $\left.l)^{2} x_{2}^{2}\right]$ so $\mathbf{H}_{1}$ is positive definite when $\lambda>0$. Therefore, when $\lambda>0$, by considering the first-order condition, we can obtain $\left(F^{*}, \tilde{p}^{*}\right)=\left(\frac{v}{2}, \frac{r+w}{2}-\frac{1}{2 \lambda}\right)$. And by checking the constraint, we can obtain that $\left(F^{*}, \tilde{p}^{*}\right)=\left(\frac{v}{2}, \frac{r+w}{2}-\frac{1}{2 \lambda}\right)$ is the optimal solution of the Lagrangian relaxation problem when $\lambda>0$ and it satisfies $-\frac{v}{h}-(r-w) \leq \frac{1}{\lambda} \leq \min \left\{\frac{v}{h}-(r-w), r+w\right\}$. By substituting $\left(F^{*}, \tilde{p}^{*}\right)$ into the objective function, we obtain the Lagrangian dual problem:

$$
\begin{aligned}
L^{*}=\max _{\lambda>0} L(\lambda) & =-Q_{c 1}\left(F^{*}, \tilde{p}^{*}\right)+\lambda \cdot\left(0-\Pi_{c 1}\left(F^{*}, \tilde{p}^{*}\right)\right) \\
\text { s.t. } & \frac{1}{\lambda} \leq \min \left\{\frac{v}{h}-(r-w), r+w\right\}, \\
& \frac{1}{\lambda} \geq-\frac{v}{h}-(r-w) .
\end{aligned}
$$

Under $v \geq h \cdot|r-w|$, coop B can only enter the market when $k \leq \tau \equiv \frac{1}{4 v} \cdot\left[(1-\alpha)(v+l(r-w))^{2}+\right.$ $\left.\alpha(v+h(r-w))^{2}\right]$. Also, under $v \geq h \cdot|r-w|$, we have $-\frac{v}{h}-(r-w) \leq 0$. By considering the first-order condition, we obtain $\lambda^{*}=\sqrt{\frac{l^{2}(1-\alpha)+h^{2} \alpha}{4 v(\tau-k)}}$. By checking the feasibility of $\lambda^{*}$, we obtain the following: 1. If $v \geq 2 r h$ so that $\frac{v}{h}-(r-w) \geq r+w$, then $\lambda^{*}$ is feasible when $k \geq \tau-\frac{(r+w)^{2}\left(l^{2}(1-\alpha)+h^{2} \alpha\right)}{4 v}$. 2. If $v<2 r h$ so that $\frac{v}{h}-(r-w)<r+w$, then $\lambda^{*}$ is feasible when $k \geq \tau-\frac{\left(\frac{v}{h}+w-r\right)^{2}\left(l^{2}(1-\alpha)+h^{2} \alpha\right)}{4 v}$. As such, if $k>\tilde{\tau} \equiv \max \left\{\tau-\frac{(r+w)^{2}\left(l^{2}(1-\alpha)+h^{2} \alpha\right)}{4 v}, \tau-\frac{\left(\frac{v}{h}+w-r\right)^{2}\left(l^{2}(1-\alpha)+h^{2} \alpha\right)}{4 v}\right\}$ so that $\lambda^{*}$ is feasible, we can check that it satisfies the complimentary slackness condition as the corresponding $\Pi_{c 1}\left(F^{*}, \tilde{p}^{*}\right)=$ 0 . By defining $\Delta^{*}=\sqrt{\frac{v(\tau-k)}{l^{2}(1-\alpha)+h^{2} \alpha}}$, we obtain the optimal solution of coop B as $\left(F_{1}^{B}, \tilde{p}_{1}^{B}\right)=\left(\frac{v}{2}, \frac{r+w}{2}-\right.$ $\left.\Delta^{*}\right)$ and the corresponding $Q_{c 1}^{B}=\frac{\alpha}{2 v}\left[v+h\left(r-w+2 \Delta^{*}\right)\right] \cdot h+\frac{1-\alpha}{v} \cdot\left[v+l\left(r-w+2 \Delta^{*}\right)\right] \cdot l$ and $\Pi_{c 1}^{B}=0$. Next, if $k \leq \tilde{\tau}$, by using Lagrangian relaxation, we obtain the corner solutions of coop B's optimal pricing problem under strategy 1 as follows. For ease of exposition, we define two thresholds $\tilde{\tau}_{0}=(\alpha h+(1-\alpha) l) \cdot(r-w)$ and $\tilde{\tau}_{1}^{\prime}=\frac{-h^{2} r^{2}(1-\alpha)-l(l r+v) w(1-\alpha)+h l r(r+w)(1-\alpha)+h v(r-w \alpha)}{v}$. 1. Suppose $\tilde{\tau}=\tilde{\tau}_{2}=\tau-\frac{\left(\frac{v}{h}+w-r\right)^{2}\left(l^{2}(1-\alpha)+h^{2} \alpha\right)}{4 v}$.

(a) If $k \in\left(\tilde{\tau}_{0}, \tilde{\tau}\right]$, then the optimal $\left(F_{1}^{B}, \tilde{p}_{1}^{B}\right)$ satisfies $\Pi_{c 1}\left(F_{1}^{B}, \tilde{p}_{1}^{B}\right)=0$ and $F_{1}^{B}+\left(\tilde{p}_{1}^{B}-r\right) \cdot h=0$ so that coop $\mathrm{B}$ can capture the entire high-consumption consumer market.

(b) If $k \leq \tilde{\tau}_{0}$, then the optimal $\left(F_{1}^{B}, \tilde{p}_{1}^{B}\right)=(0, r)$ so that coop B can capture the entire high- and low-consumption consumer markets. 
2. Suppose $\tilde{\tau}=\tilde{\tau}_{1}=\tau-\frac{(r+w)^{2}\left(l^{2}(1-\alpha)+h^{2} \alpha\right)}{4 v}$.

(a) If $k \in\left(\tilde{\tau}_{1}^{\prime}, \tilde{\tau}\right]$, then the optimal $\left(F_{1}^{B}, \tilde{p}_{1}^{B}\right)$ satisfies $\Pi_{c 1}\left(F_{1}^{B}, \tilde{p}_{1}^{B}\right)=0$ and $\tilde{p}_{1}^{B}=0$ so that coop B sets the zero member-only price.

(b) If $k \in\left(\tilde{\tau}_{0}, \tilde{\tau}_{1}^{\prime}\right]$, then the optimal $\left(F_{1}^{B}, \tilde{p}_{1}^{B}\right)$ satisfies $\Pi_{c 1}\left(F_{1}^{B}, \tilde{p}_{1}^{B}\right)=0$ and $F_{1}^{B}+\left(\tilde{p}_{1}^{B}-r\right) \cdot h=0$ so that coop B can capture the entire high-consumption consumer market.

(c) If $k \leq \tilde{\tau}_{0}$, then the optimal $\left(F_{1}^{B}, \tilde{p}_{1}^{B}\right)=(0, r)$ so that coop B can capture the entire high- and low-consumption consumer markets.

Pricing strategy 2: As maximizing $Q_{c 2}$ is equivalent to minimizing $-Q_{c 2}$, we can write the Lagrangian relaxation of coop B's problem under strategy 2 as:

$$
\begin{aligned}
L(\lambda)=\min _{\tilde{F}, p}-Q_{c 2}(\tilde{F}, p)+\lambda \cdot\left(0-\Pi_{c 2}(\tilde{F}, p)\right) & \text { s.t. }(17),(18),(23),(24),(25) .
\end{aligned}
$$

The Hessian matrix of the objective function is $\mathbf{H}_{2}=\left[\begin{array}{cc}\frac{2 \alpha \lambda}{v} & \frac{2 \lambda h \alpha}{v} \\ \frac{2 \lambda h \alpha}{v} & \frac{2 \lambda\left(h^{2} \alpha+l^{2}(1-\alpha)\right)}{v}\end{array}\right]$. For any given vector $\mathbf{x}=\left[x_{1}, x_{2}\right]^{T} \in \mathbb{R}_{++}^{2}$, we can check that $\mathbf{x}^{T} \mathbf{H}_{2} \mathbf{x}=\frac{2 \lambda}{v}\left[\alpha\left(x_{1}+h x_{2}\right)^{2}+l^{2}(1-\alpha) x_{2}^{2}\right]$ so that $\mathbf{H}_{2}$ is positive definite when $\lambda>0$. Therefore, when $\lambda>0$, by considering the first-order condition, we can obtain $\left(\tilde{F}^{*}, p^{*}\right)=\left(-\frac{(h-l) v}{2 l}, \frac{v+l(r+w)}{2 l}-\frac{1}{2 \lambda}\right)$. By checking the constraint, we can obtain that $\left(\tilde{F}^{*}, p^{*}\right)=$ $\left(-\frac{(h-l) v}{2 l}, \frac{v+l(r+w)}{2 l}-\frac{1}{2 \lambda}\right)$ is the optimal solution of the Lagrangian Relaxation problem when $\lambda>0$ and it satisfies $-\frac{v}{h}-(r-w) \leq \frac{1}{\lambda} \leq \min \left\{\frac{v}{h}-(r-w), r+w\right\}$. By substituting $\left(\tilde{F}^{*}, p^{*}\right)$ into the objective function, we obtain the following Lagrangian Dual Problem:

$$
\begin{aligned}
L^{*}=\max _{\lambda>0} L(\lambda) & =-Q_{c 2}\left(\tilde{F}^{*}, p^{*}\right)+\lambda \cdot\left(0-\Pi_{c 2}\left(\tilde{F}^{*}, p^{*}\right)\right) \\
\text { s.t. } & \frac{1}{\lambda} \leq \min \left\{\frac{v}{h}-(r-w), r+w\right\}, \\
& \frac{1}{\lambda} \geq-\frac{v}{h}-(r-w) .
\end{aligned}
$$

Under $v \geq h \cdot|r-w|$, we have $-\frac{v}{h}-(r-w) \leq 0<\frac{1}{\lambda}$ so the second constraint always holds. By considering the first-order condition, we obtain $\lambda^{*}=\sqrt{\frac{l^{2}(1-\alpha)+h^{2} \alpha}{4 v(\tau-k)}}$, which is feasible when $k \geq \tilde{\tau}$. As the corresponding $\Pi_{c 2}\left(\tilde{F}^{*}, p^{*}\right)=0$, it satisfies the complimentary slackness condition. By defining $\Delta^{*}=\sqrt{\frac{v(\tau-k)}{l^{2}(1-\alpha)+h^{2} \alpha}}$, we obtain the coop B's optimal solution as $\left(\tilde{F}^{*}, p^{*}\right)=\left(-\frac{(h-l) v}{2 l}, \frac{v+l(r+w)}{2 l}-\Delta^{*}\right)$ when $v \geq h \cdot|r-w|$ and $k \in(\tilde{\tau}, \tau]$. Thus, the coop B's optimal sales quantity $Q_{c 2}^{B}$ under pricing strategy 2 equals $Q_{c 1}^{B}$ under pricing strategy 1 . Akin to the analysis of strategy 1, we can obtain the corner solutions of coop B's problem under strategy 2 when $k \leq \tilde{\tau}$. Also, we can check that by adopting strategy 2, coop B can achieve the same optimal sales quantity as strategy 1.

Pricing strategy 3: From (10) and (12), coop B solves:

$$
Q_{c 3}^{B}=\max _{p \geq w} Q_{c 3}=\frac{\alpha}{v} \cdot[v-(p-r) \cdot h] \cdot h+\frac{1-\alpha}{v} \cdot[v-(p-r) \cdot l] \cdot l
$$




$$
\begin{array}{ll}
\text { s.t. } & (24),(26),(41), \\
& \Pi_{c 3}=\frac{\alpha}{v} \cdot[v-(p-r) \cdot h] \cdot(p-w) h+\frac{1-\alpha}{v} \cdot[v-(p-r) \cdot l] \cdot(p-w) l-k \geq 0 .
\end{array}
$$

First, from the analysis of coop A's pricing strategy 3 in the proof of Proposition 1, the coop's profit cannot exceed $\Pi_{c 3}^{A}$, where $\Pi_{c 3}^{A} \leq \tau-k$. Also, it is easy to check that the objective function is increasing in $p$ in a linear fashion, so it is optimal for coop $\mathrm{B}$ to set the optimal $p_{3}^{B}$ as small as possible. In pricing strategy $3,(F, \delta)$ are no longer the relevant decisions. Therefore, by setting $(F, \delta, p)=\left(0,0, p_{3}^{B}\right)$, we can check that it is also a feasible solution of pricing strategy 1 and 2 . Therefore, pricing strategy 3 is dominated by strategy 1 and 2. Q.E.D.

Proof of Lemma 4. From the analysis of coop B's pricing strategy 1 in the proof of Proposition 3 , we know that when $v \geq|r-w| \cdot h$ and $k \in(\tilde{\tau}, \tau]$, the optimal $\left(F_{1}^{B}, \tilde{p}_{1}^{B}\right)=\left(\frac{v}{2}, \frac{r+w}{2}-\Delta^{*}\right)$ so that the corresponding $\left(F_{1}^{B}, p_{1}^{B}, \delta_{1}^{B}\right)$ are as in Lemma 4 . By substituting $\left(F_{1}^{B}, p_{1}^{B}, \delta_{1}^{B}\right)$ into $Q_{c 1}$ as given by (2) and $\Pi_{c 1}$ as given by (4), we obtain the corresponding $Q_{c 1}^{B}$ and $\Pi_{c 1}^{B}$ as in Lemma 4. Q.E.D.

Proof of Lemma 5. From the analysis of coop B's pricing strategy 2 in the proof of Proposition 3 , we know that when $v \geq|r-w| \cdot h$ and $k \in(\tilde{\tau}, \tau]$, the optimal $\left(\tilde{F}_{2}^{B}, p_{2}^{B}\right)=\left(-\frac{(h-l) v}{2 l}, \frac{r+w}{2}+\frac{v}{2 l}-\Delta^{*}\right)$ so that we can obtain the $\left(F_{2}^{B}, p_{2}^{B}, \delta_{2}^{B}\right)$ in Lemma 5 . By substituting $\left(F_{2}^{B}, p_{2}^{B}, \delta_{2}^{B}\right)$ into $Q_{c 2}$ as given by (6) and $\Pi_{c 2}$ as given by (8), we can obtain the corresponding $Q_{c 2}^{B}$ and $\Pi_{c 2}^{B}$ in Lemma 5. Q.E.D.

Proof of Lemma 6. See the analysis of pricing strategy 3 in the proof of Proposition 3. Q.E.D. Proof of Proposition 4. In the proof of Proposition 3, we show that pricing strategy 3 is dominated by pricing strategy 1 and 2. Thus, the result follows from Lemma 4 and 5. Q.E.D.

Proof of Corollary 2. We can obtain the first and the second statements of Corollary 2 from Propositions 2 and 4 . Because $\Delta^{*} \geq 0$, it is easy to check that $Q_{r}^{A} \geq Q_{r}^{B}$ so that $Q_{r 0}-Q_{r}^{B} \geq Q_{r 0}-Q_{r}^{A}$ and $\Pi_{r 0}-\Pi_{r}^{B} \geq \Pi_{r 0}-\Pi_{r}^{A}$. Q.E.D.

Proof of Proposition 5. (a) Because $\Pi_{c}^{A}(r)$ is increasing in $r$, the coop's profit $\Pi_{c}^{A}(r) \geq \Pi_{c}^{A}\left(w_{r}\right)=$ $k_{0}-k$. Therefore, when $k \leq k_{0}$, the retailer cannot deter the coop's entry.

(b) Because the objective function (34) is increasing in $r$, the retailer should set the optimal $r$ as large as possible. Thus, when $k>k_{0}$, the supremum for the retailer's price when deterring the coop is the deterrence price $r^{D}$ that solves $\Pi_{c}^{A}\left(r^{D}\right)=0$. By solving $\Pi_{c}^{A}(r)=0$ from (33), we can derive $r^{D}$ as presented in Proposition 5 and the corresponding $\Pi_{r}^{D}$ by plugging $r^{D}$ in $\Pi_{r 0}$. Q.E.D.

Proof of Corollary 3. From the expression of $r^{D}$ as given by (35), we can check that:

$$
\frac{\partial r^{D}}{\partial k}= \begin{cases}\frac{2 v}{\sqrt{v\left[4 k\left(l^{2}(1-\alpha)+h \alpha\right)-v(h-l)^{2}(1-\alpha) \alpha\right]}} & k \in\left(k_{0}, k_{1}\right] \\ \frac{v}{\sqrt{v\left(k l^{2}(1-\alpha)+h v \alpha(l(1-\alpha)+h \alpha)\right)}} & k \in\left[k_{1}, k_{2}\right], \\ \frac{1}{l(1-\alpha)+h \alpha} & k \geq k_{2}\end{cases}
$$


so that $\frac{\partial r^{D}}{\partial k}>0$. Also, as $\Pi_{r}^{D}=\left(r^{D}-w_{r}\right) \cdot(\alpha h+(1-\alpha) l)$, we have $\frac{\partial \Pi_{r}^{D}}{\partial k}=(\alpha h+(1-\alpha) l) \cdot \frac{\partial r^{D}}{\partial k}>0$. Therefore, we have shown that both $r^{D}$ and $\Pi_{r}^{D}$ are increasing in $k$. Similarly, it follows from (35) that $r^{D}$ is increasing in $w$, where $w=w_{r}+\Delta w$. Therefore, $r^{D}$ is increasing in $\Delta w$. Also, because $r^{D}$ is increasing in $\Delta w, \Pi_{r}^{D}=\left(r^{D}-w_{r}\right) \cdot(\alpha h+(1-\alpha) l)$ is also increasing in $\Delta w$. Q.E.D.

Proof of Proposition 6. Solutions to the first-order conditions of $\Pi_{r}^{A}(r)$ in the case when $r \in$ $\left[w_{r}, \frac{v}{h}+w\right]$ and $r \in\left[\frac{v}{h}+w, \frac{v}{l}+w\right]$ are $r_{1}=\frac{w+w_{r}}{2}+\frac{l(1-\alpha)+h \alpha}{2\left(l^{2}(1-\alpha)+h^{2} \alpha\right)} \cdot v$ and $r_{2}=\frac{w+w_{r}}{2}+\frac{v}{2 l}$, respectively. Therefore, when $r \in\left[w_{r}, \frac{v}{h}+w\right], \Pi_{r}^{A}(r) \leq \Pi_{r}^{A}\left(r_{1}\right)$ and when $r \in\left[\frac{v}{h}+w, \frac{v}{l}+w\right], \Pi_{r}^{A}(r) \leq \Pi_{r}^{A}\left(r_{2}\right)$. In the case when $h \geq 3 l, r_{2}=\frac{w+w_{r}}{2}+\frac{v}{2 l}$ is within the range $\left[\frac{v}{h}+w, \frac{v}{l}+w\right]$, and $\Pi_{r}^{A}(r)$ is increasing in $r$ when $r \in\left[\frac{v}{h}+w, r_{2}\right]$ and decreasing in $r$ when $r \in\left[r_{2}, \frac{v}{l}+w\right]$. Also, under the conditions that $h \geq 2 l \cdot \frac{1-\alpha}{1-2 \alpha}$ and $\left(\sqrt{\frac{(h-l)^{2}(1-\alpha)}{h^{2} \alpha+l^{2}(1-\alpha)}}-1\right) \cdot v \geq h \cdot\left(w-w_{r}\right)$, we have $\Pi_{r}^{A}\left(r_{1}\right) \leq \Pi_{r}^{A}\left(r_{2}\right)$ so that it is optimal to set $r=r_{2}$ when $r_{2}$ is feasible. Next, by considering the constraint $\Pi_{c}^{A}(r) \geq 0$ we further derive that $r \geq r^{D}$, where $r^{D}$ is given by (35). As such, when $r^{D} \leq r_{2}$, then $r_{2}$ is feasible so it is optimal to set $r=r_{2}$; while when $r^{D}>r_{2}$, then $r_{2}$ is infeasible and $\Pi_{r}^{A}(r)$ is decreasing in $r$ so it is optimal to set $r=r^{D}$. Because $\Pi_{c}^{A}(r)$ is increasing in $r$, by solving $\Pi_{c}^{A}\left(r_{2}\right)=0$, we can further derive the threshold for $k$ as $\kappa^{T}=\Pi_{c}^{A}\left(r_{2}\right)+k$ and $\kappa^{T} \in\left[k_{1}, k_{2}\right]$ so that when $k \leq \kappa^{T}$, we have $r^{T, A}=r_{2}$ and when $k \geq \kappa^{T}$, we have $r^{T, A}=r^{D}$. It is easy to verify that both $r_{2}$ and $\Pi_{r}^{A}\left(r_{2}\right)$ are increasing in $w$ so that they are also increasing in $\Delta w$. In the proof of Corollary 3, we have already shown that $r^{D}$ is increasing in $\Delta w$. Also, it is easy to verify that when $k \in\left(\kappa^{T}, k_{2}\right]$, the corresponding $\Pi_{r}^{T, A}$ is also increasing in $\Delta w$. Q.E.D.

Proof of Proposition 7. 1. When $k>\kappa^{T}$, from Proposition 6(b) and (c), we know that if the retailer chooses to tolerate coop A's entry, then the retailer should charge $r^{T, A}=r^{D}$ so that the corresponding $\Pi_{r}^{T, A}=\Pi_{r}^{A}\left(r^{D}\right)$; while from Proposition 5, when the retailer chooses to deter the entry of coop A, then the retailer can capture the entire market by setting a slightly lower price $r^{D}-\epsilon\left(\epsilon \rightarrow 0^{+}\right)$so that $\Pi_{r}^{D} \simeq\left(r^{D}-w_{r}\right) \cdot(\alpha h+(1-\alpha) l)$. Thus, $\Pi_{r}^{T, A}=\Pi_{r}^{A}\left(r^{D}\right)<\Pi_{r}^{D}$ when $k>\kappa^{T}$.

2. Suppose $k \leq \kappa^{T}$. As $k \rightarrow k_{0}^{+}$, the deterrence price $r^{D}$ as defined Proposition 5 approaches $w_{r}$ so the corresponding profit approaches $\Pi_{r}^{D}=0$. However, if the retailer chooses to tolerate coop A's entry as $k \rightarrow k_{0}^{+}$, we can check from Proposition 7 that the retailer will set $r^{T, A}=r_{2}$ and the corresponding profit $\Pi_{r}^{T, A}>\Pi_{r}^{D}=0$. Furthermore, if the retailer chooses to tolerate coop A's entry, then its profit $\Pi_{r}^{T, A}=\frac{(1-\alpha)\left(v+l\left(w-w_{r}\right)\right)^{2}}{8 v}$ is independent of $k$, whereas $\Pi_{r}^{D}$ is increasing in $k$. Thus, there should exist a threshold $\kappa_{A} \in\left(k_{0}, \kappa^{T}\right)$ that solves $\Pi_{r}^{T, A}=\Pi_{r}^{D}(k)$ such that $\Pi_{r}^{T, A} \geq \Pi_{r}^{D}(k)$ if and only $k \leq \kappa_{A}$. Note that $\kappa_{A}$ solves $\frac{(1-\alpha)\left(v+l\left(w-w_{r}\right)\right)^{2}}{8 v}=\left(r^{D}-w_{r}\right) \cdot\left(\alpha h+(1-\alpha) l\right.$, where $r^{D}$ is as given by (35). By replacing $w$ by $w_{r}+\Delta w$, we can obtain that $\kappa_{A}$ solves $\frac{(1-\alpha)(v+l \cdot \Delta w)^{2}}{8 v(\alpha h+(1-\alpha) l)}-\Delta w=\Phi(k)$, where $\Phi(k)=r^{D}-w$. By taking the first-order derivative of the left hand side of the equation (i.e., $\left.\frac{(1-\alpha)(v+l \cdot \Delta w)^{2}}{8 v(\alpha h+(1-\alpha) l)}-\Delta w\right)$ with respect to $\Delta w$, we can observe that the value of $\frac{(1-\alpha)(v+l \cdot \Delta w)^{2}}{8 v(\alpha h+(1-\alpha) l)}-\Delta w$ is 
decreasing in $\Delta w$ when $\Delta w \in\left[-\frac{v}{h}, \frac{v}{h}\right]$. As the right hand side of the equation $\Phi(k)$ is increasing in $k$, we can obtain that $\kappa_{A}$ is decreasing in $\Delta w$. Q.E.D.

Proof pf Proposition 8. (a) As $k \rightarrow k_{0}^{+}$, the deterrence price $r^{D}$ as defined Proposition 5 approaches $w_{r}$ so the corresponding profit approaches $\Pi_{r}^{D}=0$. Thus, we will show that $\lim _{k \rightarrow k_{0}^{+}} \Pi_{r}^{T, B}>0=\lim _{k \rightarrow k_{0}^{+}} \Pi_{r}^{D}$, which - by continuity of $\Pi_{r}^{T, B}$ and $\Pi_{r}^{D}$ - implies that there exists $\kappa_{B, 1}$ such that $\Pi_{r}^{T, B}>\Pi_{r}^{D}$ for all $k \leq \kappa_{B, 1}$. Recall from $\S 4.2$ that when $k \leq(\alpha h+(1-\alpha) l) \cdot(r-w)$, it is always optimal for coop B to set $F=0$ and $p=r$ so as to capture the entire market, thus making the retailer's sales quantity $Q_{r}^{B}=0$. Note that $k_{0}-(\alpha h+(1-\alpha) l) \cdot\left(w_{r}-w\right)=\frac{1}{4 v} \cdot[(1-$ $\left.\alpha)\left(v+l\left(w_{r}-w\right)\right)^{2}+\alpha\left(v+h\left(w_{r}-w\right)\right)^{2}\right]-(\alpha h+(1-\alpha) l) \cdot\left(w_{r}-w\right)=\frac{1}{4 v} \cdot\left[(1-\alpha)\left(v-l\left(w_{r}-w\right)\right)^{2}+\right.$ $\left.\alpha\left(v-h\left(w_{r}-w\right)\right)^{2}\right]>0$. Thus, under the condition that $k \geq k_{0}>(\alpha h+(1-\alpha) l) \cdot\left(w_{r}-w\right)$, the retailer can obtain positive profits by tolerating the coop and choosing a price sufficiently close to $w_{r}$. Because the optimal tolerating profit will be higher than that, $\lim _{k \rightarrow k_{0}^{+}} \Pi_{r}^{T, B}>0=\lim _{k \rightarrow k_{0}^{+}} \Pi_{r}^{D}$. (b)-(c). As coop B's objective is to maximize its sales quantity, coop B's optimal sales quantity $Q_{c}^{B}(r) \geq Q_{c}^{A}(r)$ so that the corresponding retailer's sales quantity $Q_{r}^{B}(r) \leq Q_{r}^{A}(r)$ and retailer's profit $\Pi_{r}^{B}(r) \leq \Pi_{r}^{A}(r)$. Therefore, given the same constraint $r \geq \max \left\{r^{D}, w_{r}\right\}$, we have $\Pi_{r}^{T, B} \leq$ $\Pi_{r}^{A}\left(r^{T, B}\right) \leq \Pi_{r}^{T, A}$. Recall from Proposition EC.2, we know that when $k \geq \kappa_{A}, \Pi_{r}^{D}>\Pi_{r}^{T, A} \geq \Pi_{r}^{T, B}$, so it is optimal for the retailer to also deter the entry of coop B when $k \geq \kappa_{A}$. Thus, we can deduce two things. First, there exists $\kappa_{B, 2}$ such that $\Pi_{r}^{D}>\Pi_{r}^{T, B}$ for all $k \geq \kappa_{B, 2}$, which proves part (b). Second, because $k \geq \kappa_{A}$ is a sufficient but not necessary condition for $\Pi_{r}^{D}>\Pi_{r}^{T, B}$, by definition of $\kappa_{B, 2}$, we have $\kappa_{B, 2} \leq \kappa_{A}$, which proves part (c). Q.E.D. 


\section{Online Appendix}

\section{EC.1. Additional Analysis}

Lemma EC.1. Regardless of choosing pricing strategy 1, 2, or 3, Coop A or B can choose $(F, p, \delta)$ such that $v \geq F+(p-\delta-r) \cdot h \geq 0$ and $v \geq F+(p-\delta-r) \cdot l \geq 0$ without loss of optimality.

Proof. We will prove the result under strategy 1 but the same argument can be straightforwardly applied to strategies 2 and 3. We first show that the coop can can choose $(F, p, \delta)$ such that $F+(p-\delta-r) \cdot h \geq 0$ and $F+(p-\delta-r) \cdot l \geq 0$ without loss of optimality. First, observe that in order to have $F+(p-\delta-r) \cdot l<0$, we need $(p-\delta-r)<0$. Thus, for any policy for the coop $(F, p, \delta)$ such that $F+(p-\delta-r) \cdot l<0$, we have $F+(p-\delta-r) \cdot h<0$ as well. In such a case, the coop can improve its profit by keeping $F$ and $\delta$ the same and increasing $p$ such that $F+(p-\delta-r) \cdot l=0$, because such an increase leads to the same market share in both markets and higher profits. Consider a policy for the coop $(F, p, \delta)$ such that $F+(p-\delta-r) \cdot h<0$ and $F+(p-\delta-r) \cdot l \geq 0$. Note that under this policy, the coop captures the whole high-consumption consumer market. Consider the perturbation $(\widehat{F}, \widehat{p}, \delta)$ such that $\widehat{p}=p-\frac{(F+(p-\delta-r) \cdot h)}{h-l} \geq 0$ and $\widehat{F}=F+\frac{l(F+(p-\delta-r) \cdot h)}{h-l}=\frac{h(F+(p-\delta-r) \cdot l)}{h-l} \geq 0$. Note that this perturbation reduces $F$, so we have $\widehat{F} \leq \delta l$ (i.e., the coop still applies strategy 1). Because $\widehat{F}+(\widehat{p}-\delta-r) \cdot h=0$, after this perturbation, the coop still captures the whole high-consumption consumer market and obtains larger revenue per consumer (i.e., $F+(p-\delta) \cdot h<\widehat{F}+(\widehat{p}-\delta) \cdot h)$. Furthermore, after this perturbation, the coop's market share and revenue per consumer in the low-consumption consumer market does not change because $F+(p-\delta-r) \cdot l=\widehat{F}+(\widehat{p}-\delta-r) \cdot l$. Thus, coop A is strictly better off (i.e., has strictly larger profit) and coop B is not worse off (i.e., has the same market share) after the perturbation. Therefore, the coop can restrict attention to $(F, p, \delta)$ that satisfies $F+(p-\delta-r) \cdot h \geq 0$ and $F+(p-\delta-r) \cdot l \geq 0$ without loss of optimality.

We next show that the coop can choose $(F, p, \delta)$ such that $v \geq F+(p-\delta-r) \cdot h$ and $v \geq F+(p-$ $\delta-r) \cdot l$ without loss of optimality under the assumption that $(p-\delta-r) \geq 0$, but our argument can straightforwardly applied to the case where $(p-\delta-r)<0$. Under any policy for the coop $(F, p, \delta)$ such that $v-(F+(p-\delta-r) \cdot l)<0$, we also have $v-(F+(p-\delta-r) \cdot h) \leq v-(F+(p-\delta-r) \cdot l)<0$ as well. In such a case, the coop has zero market share. Thus, restricting attention to $(F, p, \delta)$ such that $v-(F+(p-\delta-r) \cdot h) \geq 0$ and $v-(F+(p-\delta-r) \cdot l) \geq 0$ is without loss of optimality because $(F, p, \delta)=(v, r, 0)$ satisfies these conditions and can obtain zero market share. Consider a policy for the coop $(F, p, \delta)$ such that $v-(F+(p-\delta-r) \cdot h)<0 \leq v-(F+(p-\delta-r) \cdot l)<$ 0 . Note that under this policy, the coop captures zero market share in the high-consumption consumer market. Consider the perturbation $(\widehat{F}, p, \widehat{\delta})$ such that $\widehat{\delta}=\delta-\frac{v-(F+(p-\delta-r) \cdot h)}{h-l} \geq 0$ and $\widehat{F}=F-\frac{l(v-(F+(p-\delta-r) \cdot h))}{h-l} \geq 0$. Note that this perturbation ensures that $\widehat{F} \leq \widehat{\delta} l$ (i.e., the coop still 
applies strategy 1). After the perturbation, $v-(\widehat{F}+(p-\widehat{\delta}-r) \cdot h)=0$ and $v-(\widehat{F}+(p-\widehat{\delta}-r) \cdot l)=$ $v-(F+(p-\delta-r) \cdot l) \geq 0$. (Note that we have $v-(\widehat{F}+(p-\widehat{\delta}-r) \cdot h) \leq v-(\widehat{F}+(p-\widehat{\delta}-r) \cdot l)$, which implies that $p-\widehat{\delta} \geq r>0$.) Thus, by implementing $(\widehat{F}, p, \widehat{\delta})$ instead of $(F, p, \delta)$, the coop obtains the same market share, the same (zero) profit from the high-consumption consumer market, and the same profit from the low-consumption market because $\widehat{F}+(p-\widehat{\delta}-r) \cdot l=F+(p-\delta-r) \cdot l$. Therefore, because $(\widehat{F}, p, \widehat{\delta})$ also satisfies $v-(\widehat{F}+(p-\widehat{\delta}-r) \cdot h) \geq 0$ and $v-(\widehat{F}+(p-\widehat{\delta}-r) \cdot l) \geq 0$, restricting attention to policies that satisfy these conditions is without loss of optimality.

\section{EC.1.1. Sales and Profit of Different Stores When the Coop Adopts Pricing Strategy 2}

When the coop sets $F \in[\delta l, \delta h]$ under strategy 2, only high-consumption coop shoppers will become members. By noting the consumers have to pay $r$ per unit when shopping at the retailer, (1) low-consumption consumers with annual consumption $l$ will shop at the coop if $p l-S \leq r l$ and shop at the retailer if $p l-S \geq r l$; and (2) high-consumption consumers with annual consumption $h$ will shop at the coop if $F+(p-\delta) h-S \leq r h$ and shop at the retailer if $F+(p-\delta) h-S \geq r h$. Because $S \sim U[0, v]$, the proportion of high-consumption consumers that buy from the coop and the retailer can be written as $\min \{\max \{[v-(F+(p-\delta-r) \cdot h)] / v, 0\}, 1\}$ and $\min \{\max \{[F+(p-$ $\delta-r) \cdot h] / v, 0\}, 1\}$, respectively; and the proportion of low-consumption consumers that buy from the coop and the retailer can be written as $\min \{\max \{[v-(p-r) \cdot l] / v, 0\}, 1\}$ and $\min \{\max \{[(p-r)$. $l] / v, 0\}, 1\}$,respectively. By Lemma EC.1, coop A or B can choose $(F, p, \delta)$ such that $v \geq F+(p-$ $\delta-r) \cdot h \geq 0$ and $p-r \cdot l \geq 0$ without loss of optimality. As the proportion of high-consumption type is $\alpha$, we can determine the sales quantities for each store when the coop adopts pricing strategy 2 as depicted in Table EC.1.

Table EC.1 Sales quantities at different stores when the coop adopts pricing strategy 2 .

\begin{tabular}{c|c|c}
\hline & retailer & food coop \\
\hline high-consumption type & $\frac{\alpha}{v} \cdot[F+(p-\delta-r) \cdot h] \cdot h$ & $\frac{\alpha}{v} \cdot[v-(F+(p-\delta-r) \cdot h)] \cdot h$ \\
low-consumption type & $\frac{1-\alpha}{v} \cdot(p-r) \cdot l \cdot l$ & $\frac{1-\alpha}{v} \cdot(v-(p-r) \cdot l) \cdot l$ \\
\hline
\end{tabular}

Given the sales quantities in Table EC.1, the retailer's unit cost $w_{r}$, and the coop's unit cost $w$, we can derive the retailer's sales quantity $Q_{r 2}$, the coop's sales quantity $Q_{c 2}$, and the corresponding retailer profit $\Pi_{r 2}$ and coop profit $\Pi_{c 1}$ as given by (5), (6), (7), and (8).

\section{EC.1.2. Sales and Profit of Different Stores When the Coop Adopts Pricing Strategy 3}

When the coop sets $F \geq \delta h$ under strategy 3, no coop shoppers will become member. By noting that consumer has to pay $r$ per unit when shopping at the retailer, consumers with annual consumption $j(\in\{h, l\})$ will shop at the coop if $p \cdot j-S \leq r \cdot j$, and shop at the retailer if $p \cdot j-S \geq r \cdot j$. Because $S \sim U[0, v]$, the proportion of consumers of type $j$ that buy from the coop and the retailer can be written as $\min \{\max \{[v-(p-r) \cdot j] / v, 0\}, 1\}$ and $\min \{\max \{[(p-r) \cdot j] / v, 0\}, 1\}$, respectively. By 
Lemma EC.1, the coop can restrict attention to $p$ such that $p-r \geq 0$ for all $j(\in\{h, l\})$ without loss of optimality. Because the proportion of high-consumption type is $\alpha$, we can determine the sales quantities for each store when the coop adopts pricing strategy 3 in Table EC.2.

Table EC.2 Sales quantities at different stores when the coop adopts pricing strategy 3 .

\begin{tabular}{c|c|c}
\hline & retailer & food coop \\
\hline high-consumption type & $\frac{\alpha}{v} \cdot(p-r) \cdot h \cdot h$ & $\frac{\alpha}{v} \cdot(v-(p-r) \cdot h) \cdot h$ \\
low-consumption type & $\frac{1-\alpha}{v} \cdot(p-r) \cdot l \cdot l$ & $\frac{1-\alpha}{v} \cdot(v-(p-r) \cdot l) \cdot l$ \\
\hline
\end{tabular}

Given the sales quantities in Table EC.2, the retailer's unit cost $w_{r}$, and the coop's unit cost $w$, we can derive the retailer's sales quantity $Q_{r 3}$, the coop's sales quantity $Q_{c 3}$, and the corresponding retailer profit $\Pi_{r 3}$ and coop profit $\Pi_{c 3}$ as given by (9), (10), (11), and (12).

\section{EC.1.3. Deterrence Strategy against Coop A's Potential Entry}

Before we determine the optimal solution of the retailer's tolerating problem (37), we first use the following lemma to show the different structures of $\Pi_{r}^{A}(r)$ as given by (36) under different cases where the parameters satisfy different conditions.

Lemma EC.2. Upon coop A's entry, the structure of the retailer's profit $\Pi_{r}^{A}(r)$ is as follows:

(a) If $w \in\left[w_{r}+\left(\frac{1}{l}-\frac{2}{h}\right) v, w_{r}+\frac{v}{h}\right], \Pi_{r}^{A}(r)$ is increasing in $r$ when $r \in\left[w_{r}, r_{1}\right]$, and decreasing in $r$ when $r \in\left[r_{1}, \frac{v}{l}+w\right]$.

(b) If $w \in\left(w_{r}+\frac{v}{h} \cdot\left(\sqrt{\frac{(h-l)^{2}(1-\alpha)}{h^{2} \alpha+l^{2}(1-\alpha)}}-1\right), w_{r}+\left(\frac{1}{l}-\frac{2}{h}\right) v\right), \Pi_{r}^{A}(r)$ is increasing in $r$ when $r \in\left[w_{r}, r_{1}\right]$, decreasing in $r$ when $r \in\left[r_{1}, \frac{v}{h}+w\right]$, increasing in $r$ when $r \in\left[\frac{v}{h}+w, r_{2}\right]$, and decreasing in $r$ when $r \in\left[r_{2}, \frac{v}{l}+w\right]$. Furthermore, $\Pi_{r}^{A}\left(r_{1}\right) \geq \Pi_{r}^{A}\left(r_{2}\right)$.

(c) If $w \in\left[w_{r}+\left(\frac{l(1-\alpha)+h \alpha}{l^{2}(1-\alpha)+h^{2} \alpha}-\frac{2}{h}\right) v, w_{r}+\frac{v}{h} \cdot\left(\sqrt{\frac{(h-l)^{2}(1-\alpha)}{h^{2} \alpha+l^{2}(1-\alpha)}}-1\right)\right), \Pi_{r}^{A}(r)$ is increasing in $r$ when $r \in$ $\left[w_{r}, r_{1}\right]$, decreasing in $r$ when $r \in\left[r_{1}, \frac{v}{h}+w\right]$, increasing in $r$ when $r \in\left[\frac{v}{h}+w, r_{2}\right]$, and decreasing in $r$ when $r \in\left[r_{2}, \frac{v}{l}+w\right]$. Furthermore, $\Pi_{r}^{A}\left(r_{1}\right)<\Pi_{r}^{A}\left(r_{2}\right)$.

(d) If $w \in\left[w_{r}-\frac{v}{h}, w_{r}+\left(\frac{l(1-\alpha)+h \alpha}{l^{2}(1-\alpha)+h^{2} \alpha}-\frac{2}{h}\right) v\right), \Pi_{r}^{A}(r)$ is increasing in $r$ when $r \in\left[w_{r}, r_{2}\right]$ and decreasing in $r$ when $r \in\left[r_{2}, \frac{v}{l}+w\right]$.

Proof of Lemma EC.2. From (36), we know that

- When $w_{r} \leq r \leq \frac{v}{h}+w, \Pi_{r}^{A}(r)=\left(r-w_{r}\right) \cdot\left[\frac{h \alpha}{2 v}(v-h(r-w))+\frac{l(1-\alpha)}{2 v}(v-l(r-w))\right]$ and by using the first-order condition, we obtain $r_{1}=\frac{w+w_{r}}{2}+\frac{l(1-\alpha)+h \alpha}{2\left(l^{2}(1-\alpha)+h^{2} \alpha\right)} \cdot v$ so that when $r<r_{1}, \Pi_{r}^{A}(r)$ is increasing in $r$; whereas when $r>r_{1}, \Pi_{r}^{A}(r)$ is decreasing in $r$.

- When $\frac{v}{h}+w \leq r \leq \frac{v}{l}+w, \Pi_{r}^{A}(r)=\left(r-w_{r}\right) \cdot \frac{l(1-\alpha)}{2 v}(v-l(r-w))$ and by using the first-order condition, we obtain $r_{2}=\frac{w+w_{r}}{2}+\frac{v}{2 l}$ so that when $r \leq r_{2}, \Pi_{r}^{A}(r)$ is increasing in $r$; whereas when $r>r_{2}, \Pi_{r}^{A}(r)$ is decreasing in $r$.

- When $r \geq \frac{v}{l}+w, \Pi_{r}^{A}(r)=0$. 
Next, by checking whether $r_{1}$ is within the range $\left[w_{r}, \frac{v}{h}+w\right]$ and whether $r_{2}$ is within the range $\left[\frac{v}{h}+w, \frac{v}{l}+w\right]$ and by comparing $\Pi_{r}^{A}\left(r_{1}\right)$ and $\Pi_{r}^{A}\left(r_{2}\right)$, we can obtain the structure of $\Pi_{r}^{A}(r)$ under different cases as given by Lemma EC.2. Q.E.D.

Based on the results shown in Lemma EC.2, the profit of the retailer $\Pi_{r}^{A}(r)$ can take the four different shapes illustrated in Figure EC.1.

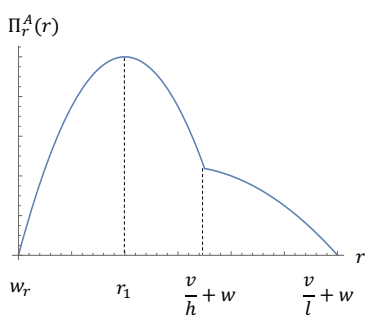

(a)

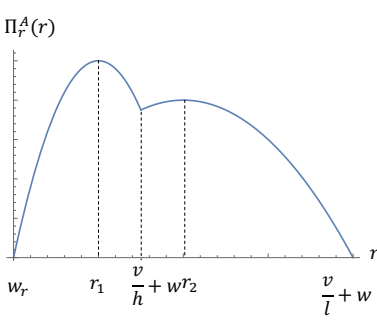

(b)

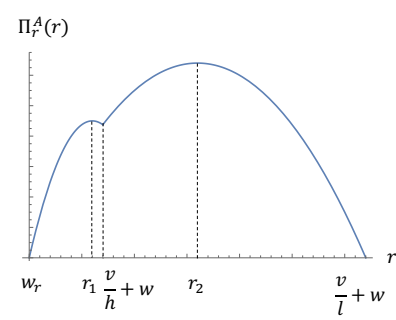

(c)

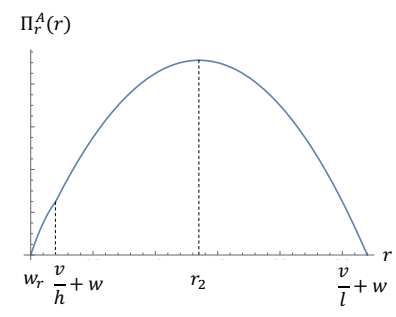

(d)

Figure EC.1 The structure of $\Pi_{r}^{A}(r)$ under different cases.

As in $\S 5.1$, because $\Pi_{c}^{A}(r)$ is increasing in the retailer's price $r$, the constraint $\Pi_{c}^{A}(r) \geq 0$ holds if and only the optimal $r$ that solves (37) satisfies $r \geq r^{D}$, where $r^{D}$ is characterized by (35). Next, based on the structure of the objective function $\Pi_{r}^{A}(r)$ and by checking the feasible region of $r$, we can derive the optimal solution of (37) under different cases as given by Proposition EC.1. In preparation and for ease of exposition, we define $\tilde{\Pi}_{c}^{A}(r) \equiv \Pi_{c}^{A}(r)+k$ as the coop's "gross" profit before deducting the fixed annual operating cost $k$. Notice that that $\tilde{\Pi}_{c}^{A}(r)$ is independent of $k$.

Proposition EC.1. When the retailer tolerates coop A's entry, its optimal price $r^{T, A}$ can be characterized as follows:

(a) Suppose $w \in\left[w_{r}+\left(\frac{1}{l}-\frac{2}{h}\right) v, w_{r}+\frac{v}{h}\right]$.

1. If $k \leq \tilde{\Pi}_{c}^{A}\left(r_{1}\right)$, then $r^{T, A}=r_{1}$ so that $\Pi_{r}^{T, A}=\Pi_{r}^{A}\left(r_{1}\right)$;

2. If $k>\tilde{\Pi}_{c}^{A}\left(r_{1}\right)$, then $r^{T, A}=r^{D}$ so that $\Pi_{r}^{T, A}=\Pi_{r}^{A}\left(r^{D}\right)$.

(b) Suppose $w \in\left(w_{r}+\frac{v}{h} \cdot\left(\sqrt{\frac{(h-l)^{2}(1-\alpha)}{h^{2} \alpha+l^{2}(1-\alpha)}}-1\right), w_{r}+\left(\frac{1}{l}-\frac{2}{h}\right) v\right)$. Then there exists $r_{3} \in\left[r_{1}, \frac{v}{h}+w\right]$ such that $\Pi_{r}^{A}\left(r_{3}\right)=\Pi_{r}^{A}\left(r_{2}\right)$.

1. If $k \leq \tilde{\Pi}_{c}^{A}\left(r_{1}\right)$, then $r^{T, A}=r_{1}$ and $\Pi_{r}^{T, A}=\Pi_{r}^{A}\left(r_{1}\right)$;

2. If $k \in\left(\tilde{\Pi}_{c}^{A}\left(r_{1}\right), \tilde{\Pi}_{c}^{A}\left(r_{3}\right)\right)$, then $r^{T, A}=r^{D}$ and $\Pi_{r}^{T, A}=\Pi_{r}^{A}\left(r^{D}\right)$;

3. If $k \in\left[\tilde{\Pi}_{c}^{A}\left(r_{3}\right), \tilde{\Pi}_{c}^{A}\left(r_{2}\right)\right]$, then $r^{T, A}=r_{2}$ and $\Pi_{r}^{T, A}=\Pi_{r}^{A}\left(r_{2}\right)$;

4. If $k>\tilde{\Pi}_{c}^{T, A}\left(r_{2}\right)$, then $r^{T, A}=r^{D}$ and $\Pi_{r}^{T, A}=\Pi_{r}^{A}\left(r^{D}\right)$.

(c) Suppose $w \in\left[w_{r}-\frac{v}{h}, w_{r}+\frac{v}{h} \cdot\left(\sqrt{\frac{(h-l)^{2}(1-\alpha)}{h^{2} \alpha+l^{2}(1-\alpha)}}-1\right)\right)$.

1. If $k \leq \tilde{\Pi}_{c}^{A}\left(r_{2}\right)$, then $r^{T, A}=r_{2}$ so that $\Pi_{r}^{T, A}=\Pi_{r}^{A}\left(r_{2}\right)$;

2. If $k>\tilde{\Pi}_{c}^{A}\left(r_{2}\right)$, then $r^{T, A}=r^{D}$ so that $\Pi_{r}^{T, A}=\Pi_{r}^{A}\left(r^{D}\right)$. 
Proof of Proposition EC.1. According to the property of $\Pi_{r}^{A}(r)$ as characterized by Lemma EC.2, we can solve (37) by analyzing the following cases:

(a) When $w \in\left[w_{r}+\left(\frac{1}{l}-\frac{2}{h}\right) v, w_{r}+\frac{v}{h}\right]$ (Lemma EC.2 (a)), to maximize $\Pi_{r}^{A}(r)$, it is optimal to set $r^{T, A}=r_{1}$ when $r_{1}$ is feasible. By checking the feasibility of $r_{1}$, we know that when $k \leq \tilde{\Pi}_{c}^{A}\left(r_{1}\right)$ so that $r_{1} \geq r^{D}$, it is optimal to set $r^{T, A}=r_{1}$; else when $k>\tilde{\Pi}_{c}^{A}\left(r_{1}\right)$ so that $r_{1}$ is no longer feasible, it is optimal to set $r^{T, A}=r^{D}$ as $\Pi_{r}^{A}(r)$ is decreasing in $r$ when $r \geq r_{1}$.

(b) When $w \in\left(w_{r}+\frac{v}{h}\left(\sqrt{\frac{(h-l)^{2}(1-\alpha)}{h^{2} \alpha+l^{2}(1-\alpha)}}-1\right), w_{r}+\left(\frac{1}{l}-\frac{2}{h}\right) v\right)$ (Lemma EC.2 (b)), to maximize $\Pi_{r}^{A}(r)$, it is optimal to set $r^{T, A}=r_{1}$ when $r_{1}$ is feasible. When $k \leq \tilde{\Pi}_{c}^{A}\left(r_{1}\right)$ so that $r_{1} \geq r^{D}$, it is optimal to set $r^{T, A}=r_{1}$. Next, as $\Pi_{r}^{A}(r)$ is decreasing in $r$ when $r \in\left[r_{1}, \frac{v}{h}+w\right]$ and increasing in $r$ when $r \in\left[\frac{v}{h}+w, r_{2}\right]$, we can check that there exists $r_{3} \in\left[r_{1}, \frac{v}{h}+w\right]$ that satisfies $\Pi_{r}^{A}\left(r_{3}\right)=\Pi_{r}^{A}\left(r_{2}\right)$. Therefore, (1) if $k \in\left(\tilde{\Pi}_{c}^{A}\left(r_{1}\right), \tilde{\Pi}_{c}^{A}\left(r_{3}\right)\right)$ so that $r^{D} \in\left(r_{1}, r_{3}\right)$ and $\Pi_{r}^{A}\left(r^{D}\right)>\Pi_{r}^{A}\left(r_{3}\right)=\Pi_{r}^{A}\left(r_{2}\right)$, then it is optimal to set $r^{T, A}=r^{D} ;(2)$ if $k \in\left[\tilde{\Pi}_{c}^{A}\left(r_{3}\right), \tilde{\Pi}_{c}^{A}\left(r_{2}\right)\right]$ so that $r^{D} \in\left[r_{3}, r_{2}\right]$, it is optimal to set $r^{T, A}=r_{2} ;(3)$ if $k>\tilde{\Pi}_{c}^{A}\left(r_{2}\right)$ so that $r^{D} \geq r_{2}$, it is optimal to set $r^{T, A}=r^{D}$.

(c) When $w \in\left[w_{r}-\frac{v}{h}, w_{r}+\frac{v}{h}\left(\sqrt{\frac{(h-l)^{2}(1-\alpha)}{h^{2} \alpha+l^{2}(1-\alpha)}}-1\right)\right]$ (Lemma EC.2 (c) and (d)), it is optimal to set $r^{T, A}=r_{2}$ when $r_{2}$ is feasible. Therefore, when $k \leq \tilde{\Pi}_{c}^{A}\left(r_{2}\right)$ so that $r_{2} \geq r^{D}$, then it is optimal to set $r^{T, A}=r_{2}$; else when $k>\tilde{\Pi}_{c}^{A}\left(r_{2}\right)$, it is optimal to set $r^{T, A}=r^{D}$. Q.E.D.

We now determine the retailer's optimal deterrence strategy against coop A by comparing $\Pi_{r}^{D}$ and $\Pi_{r}^{T, A}$ as given by Propositions 5 and EC.1, respectively. Recall from Proposition 5 that the retailer cannot deter the coop's entry when $k \leq k_{0}=\tau\left(w_{r}\right)$. Therefore, we shall only analyze the retailer's deterrence strategy against the coop A's potential entry for the case when $k>k_{0}$.

Proposition EC.2. Suppose coop A's fixed annual operating cost $k>k_{0}$ so that the retailer is able to deter coop A's entry if so desired. Then there exists a threshold $\kappa_{A}$ such that it is optimal for the retailer to

1. Tolerate coop A's entry and set its selling price $r^{T, A}$ as described by Proposition EC.1 when $k \leq \kappa_{A} ;$

2. Deter coop A's entry and set its selling price below $r^{D}$ as described in Proposition 5 when $k>\kappa_{A}$. Also, the threshold $\kappa_{A}$ satisfies the following properties:

(a) Suppose $w \in\left[w_{r}+\frac{v}{h} \cdot\left(\sqrt{\frac{(h-l)^{2}(1-\alpha)}{h^{2} \alpha+l^{2}(1-\alpha)}}-1\right), w_{r}+\frac{v}{h}\right]$. Then $\kappa_{A} \in\left(k_{0}, \tilde{\Pi}_{c}^{A}\left(r_{1}\right)\right]$ and it satisfies $\Pi_{r}^{D}\left(\kappa_{A}\right)=\Pi_{r}^{A}\left(r_{1}\right)=\frac{\left[\alpha h\left(v+h\left(w-w_{r}\right)\right)+(1-\alpha) l\left(v+l\left(w-w_{r}\right)\right)\right]^{2}}{8 v\left(l^{2}(1-\alpha)+h^{2} \alpha\right)} ;$

(b) Suppose $w \in\left[w_{r}-\frac{v}{h}, w_{r}+\frac{v}{h} \cdot\left(\sqrt{\frac{(h-l)^{2}(1-\alpha)}{h^{2} \alpha+l^{2}(1-\alpha)}}-1\right)\right)$. Then, $\kappa_{A} \in\left(k_{0}, \tilde{\Pi}_{c}^{A}\left(r_{2}\right)\right]$ and it satisfies $\Pi_{r}^{D}\left(\kappa_{A}\right)=\Pi_{r}^{A}\left(r_{2}\right)=\frac{(1-\alpha)\left(v+l\left(w-w_{r}\right)\right)^{2}}{8 v}$.

Proof of Proposition EC.2. The proof follows using a similar approach to that we used in the proof of Proposition 7. Q.E.D. 